\title{
6 Nachwehen
}

\subsection{Nachkriegsspuren in den Anden - Fluchtort Argentinien}

Argentinien entwickelte sich nach Kriegsende 1945 erneut $\mathrm{zu}$ einem beliebten Auswanderungsland für deutsche und österreichische MigrantInnen. Allein 30000 bis 40000 Frauen und Männer deutscher Herkunft sollen in den ersten zehn Jahren nach Kriegsende nach Argentinien ausgewandert sein. ${ }^{1}$ Das hatte nicht zuletzt mit dem wirtschaftlichen Aufschwung des südamerikanischen Staates zu tun, sondern auch damit, dass der argentinische Staatspräsident Juan Perón sich explizit um deutsche Fachkräfte aus Naturwissenschaft und Technik bemühte. Diese fanden in Argentinien meist eine gut dotierte Arbeitsstelle, die ihnen zum Teil große Freiheiten einräumte. ${ }^{2}$ Wie viele von ihnen tatsächlich NationalsozialistInnen waren und nicht zuletzt NS-Kriegsverbrecher, darüber sind sich HistorikerInnen nicht ganz einig. Manche schätzen die Zahl auf bis zu $70000 .{ }^{3}$ Fakt ist, Argentinien war aus mehreren Gründen ein begehrtes Fluchtziel von ehemaligen hochrangigen NSDAP-Parteigängern sowie SAund SS-Funktionären. ${ }^{4}$

Die Regierung Perón setzte für den Aufbau der argentinischen Wirtschaft bewusst auf deutsches Know-how. Laut dem argentinischen Historiker Felix Luna dürfte Perón eine gewisse Sympathie für Nazi-Deutschland empfunden haben. Er sei fasziniert gewesen von dem „Brimborium totalitärer Regime“5, stellte Luna 1992 in einem Artikel im deutschen Nachrichten-Magazin Der Spiegel fest. Auch Philipp Mettauer verweist in seiner Studie zur jüdischen Flucht nach Argentinien auf Peróns Sympathien für den Nationalsozialismus und seine

1 Vgl. Heinz Schneppen, Odessa und das Vierte Reich. Mythen der Zeitgeschichte, Berlin 2007, S. 114.

2 Vgl. Holger M. Meding, Flucht vor Nürnberg, Deutsche und österreichische Einwanderung in Argentinien 1945-1955, Wien/Köln/Weimar 1992, S. 89 und 92.

3 Vgl. Schneppen, Odessa, S, 114.

4 Vgl. Edith Blaschitz, Austrian National Socialists in Argentina after 1945, in: Oliver Rathkolb (Hg.), Revisiting the National Socialist Legacy. Coming to Terms with Forced Labor, Expropriation, Compensation and Restitution, Innsbruck 2002, S. 226-240; Edith Blaschitz, Austrian National Socialists: The Route to Argentina, o. S., https://www.donau-uni.ac.at/dam/jcr:b3e8197fdd87-4f3e-98be-fc06236dfofe/holocausteraassets_blaschitz.pdf (7.8.2019).

5 Zit. nach Dunkle Seite des Mondes, Der Spiegel, Nr. 12, 16.3.1992, http://www.spiegel.de/ spiegel/print/d-13682067.html (9.4.2017). 
aktive Fluchthilfe für nationalsozialistische Verbrecher nach $1945 .{ }^{6}$ Perón hatte 1939 bei seinem Aufenthalt als Militärattaché in Italien und Deutschland den Faschismus, Nationalsozialismus und Antisemitismus kennengelernt. Wie andere hochrangige Militärs in Argentinien war Perón vom Sieg der Achsenmächte überzeugt und davon, dass Argentinien die Führungsrolle in Südamerika übernehmen sollte. ${ }^{7}$ Auch wenn es zu Beginn des Zweiten Weltkriegs kleinere militärische Zusammenstöße zwischen Argentinien und dem Deutschen Reich auf See gegeben hatte und die diplomatischen Beziehungen zu den Achsenmächten im Jänner 1944 abgebrochen worden waren, so wurden die informellen Kontakte sowie die seit den frühen 1930er-Jahren bestehenden nationalsozialistischen Netzwerke weiterhin gepflegt. Die späte Kriegserklärung Ende März 1945 auf Druck der Alliierten war ein Ablenkungsmanöver und gab der argentinischen Regierung Zeit für die Organisation der nationalsozialistischen Flucht nach Argentinien. ${ }^{8}$ Auf diese Weise lässt sich unter anderem erklären, warum gegen Ende der 1940er-Jahre tausende Nationalsozialisten den Fluchtweg über Italien, das Mittelmeer und den Atlantik nach Buenos Aires wählten. Doch es war nicht nur Peróns Faible für das nationalsozialistische Deutschland, der zu einem Anstieg der Auswanderung aus Nachkriegsdeutschland und -österreich Richtung Argentinien führte und die Flucht für NS-Täter und Kriegsverbrecher erleichterte. Der deutsche Historiker Holger M. Meding stellt zudem fest, dass es über das Wanderungsamt des Innenministeriums in Wien relativ einfach war, Informationen über die argentinischen Interessen an Fachkräften zu erhalten, und dass sich in Genua eine argentinische Einwanderungsmission befand. Zudem unterstützte eine Argentinisch-Österreichische Gesellschaft die Einreiseformalitäten. ${ }^{9}$

Argentinien zählte bis in die 1930er-Jahre zu einem klassischen Einwanderungsland. Neben den mehrheitlich italienischen und spanischen Einwanderern lebten zu diesem Zeitpunkt auch 240000 Deutsche, ÖsterreicherInnen und SchweizerInnen in dem südamerikanischen Land. ${ }^{10}$ Die deutsche Auswanderung in Argentinien konnte nicht nur auf eine lange Tradition zurückblicken, sie stand ebenso in enger Beziehung zum nationalsozialistischen Deutschland. Bereits im Februar 1931 war in Buenos Aires die erste NSDAP-Ortsgruppe ge-

\footnotetext{
6 Vgl. Philipp Mettauer, Erzwungene Emigration nach Argentinien. Österreichisch-jüdische Lebensgeschichten, Studien zur Geschichte und Kultur der Iberischen und Iberoamerikanischen Länder (Band 14), Münster 2010, S. 137.

7 Vgl. Mettauer, Erzwungene Emigration, S. 138.

8 Vgl. Mettauer, Erzwungene Emigration, S. 142-145.

9 Vgl. Meding, Flucht vor Nürnberg, S. 67-68.

10 Vgl. Mettauer, Erzwungene Emigration, S. 86.
} 
gründet worden. ${ }^{11} \mathrm{Zu}$ Jahresbeginn 1933 umfasste die Landesgruppe Argentinien der NSDAP 220 Mitglieder. Durch ein strenges Aufnahmeverfahren waren es zwar nie mehr als 2000 Parteimitglieder, die nationalsozialistische Ideologie dürfte aber auf die Argentiniendeutschen ausgestrahlt haben, deren Gruppe in den 1930er-Jahren bis zu 300000 Personen umfasste, schreibt Meding. ${ }^{12}$ Nikolaus Barbian verweist außerdem darauf, dass die „wichtigsten deutschen Vereine" in den 1930er-Jahren unter nationalsozialistische Kontrolle gebracht werden konnten. ${ }^{13}$ Einer dieser stark deutsch-geprägten Vereine war unter anderem der Club Andino Bariloche. Wie bereits geschildert, geht dieser noch heute existierende Alpin- und Skiclub auf den aus Bayern stammenden Auswanderer Otto Meiling zurück und wies bereits in den frühen 1930er-Jahren etliche deutsche Mitglieder auf. ${ }^{14}$ Sehr wohl war der Club Andino Bariloche kein rein deutscher Verein, sondern von Beginn von anderen europäischen AuswandererInnen geprägt, allerdings spielten deutsche und österreichische Mitglieder neben ItalienerInnen und SchweizerInnen eine tragende Rolle. ${ }^{15}$ Der Innsbrucker Skilehrer Hans Nöbl, der im Vereinsvorstand saß, zeichnete immerhin für die Gründung des Skigebietes in Bariloche verantwortlich. ${ }^{16}$

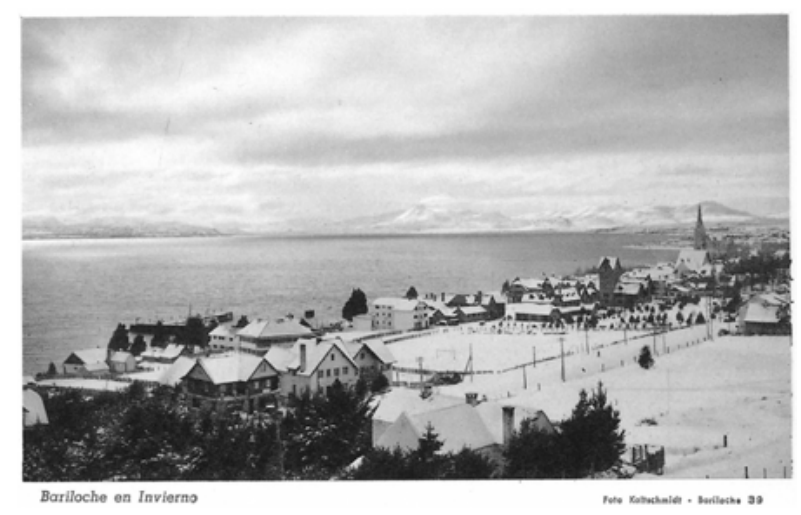

Abb. 37: Der Ski- und Wintersportort San Carlos de Bariloche mit dem See Nahuel Huapi und den Andengipfeln im Hintergrund, Kaltschmidt.

11 Vgl. Nikolaus Barbian, Auswärtige Kulturpolitik und „Auslandsdeutsche“ in Lateinamerika 1949-1973, Wiesbaden 2014, S. 94.

12 Vgl. Meding, Flucht vor Nürnberg, S. 29-30.

13 Vgl. Barbian, Auswärtige Kulturpolitik, S. 94.

14 Vgl. Arko, Otto Meiling, S. 18-19.

15 Die Herkunft der Klubmitglieder im CAB spiegelt auch die Herkunft der Einwanderer in Bariloche wider. Vgl. Hans Schulz, Bariloche, S. 57.

16 Vgl. dazu „Hans Nöbl - ein Tiroler in den argentinischen Anden“ in Kapitel 2 der Publikation. 
Mit den Fachkräften ergriffen auch österreichische Skilehrer und Skiathleten, die wegen illegaler Handlungen vor 1938 und aufgrund ihrer Betätigungen für das NS-System von der Nachkriegsjustiz verfolgt wurden, die Chance, in Argentinien ein neues Leben anzufangen. Als hilfreich erwiesen sich dabei persönliche Netzwerke, die meist schon seit der Zwischenkriegszeit bestanden. Gemeinsame sportliche Interessen brachten die Skikameraden der 1930er-Jahre in Argentinien wieder zusammen. Die Berge als Sehnsuchtsort und die Aussicht auf gemeinsame Unternehmungen in einem vertrauten, aber dennoch exotischen Terrain, machten die patagonische Pampa zu einem beliebten Fluchtziel. Die Alpen wurden gegen die Anden eingetauscht, der Kriegsschauplatz und der NS-Terror gegen eine weit entfernte, vermeintlich friedliche Natur-Idylle. ${ }^{17}$ Das belegen nicht zuletzt die Mitgliederverzeichnisse des Club Andino Bariloche der späten 1940er- und 1950er-Jahre. In diesen finden sich nicht nur die Angehörigen der belasteten Familie Lantschner, sondern auch andere ehemalige hochrangige Nationalsozialisten. Gleichzeitig wird die Verbundenheit an den bergsteigerischen Unternehmungen und bei skisportlichen Veranstaltungen sichtbar. Expeditionen zu umliegenden Andengipfeln, meist auch per Ski, standen im Club Andino Bariloche an der Tagesordnung. Die Meisterschaften im alpinen Skisport wurden auf dem nahe gelegenen Cerro Catedral ausgetragen. ${ }^{18}$ Hier zogen Fritz und Gustav Lantschner als Federico und Gustavo Lantschner ihre Schwünge und fuhren Konkurrenzen im Slalom gegen ehemalige österreichische und deutsche Nationalsozialisten, italienische Faschisten und Kommunisten, kroatische UstaschaAnhänger und politisch scheinbar neutrale Schweizer.

Sie trafen aber ebenso auf jüdische Einwandererfamilien aus halb Europa, die bereits vor der nationalsozialistischen Herrschaft ausgewandert und aufgrund der NS-Verfolgung geflüchtet waren. ${ }^{19}$ Argentinien galt als eines der „europäischsten“ Länder Südamerikas. Die Zahl der EinwohnerInnen stieg aufgrund der Einwanderung von 1930 bis 1949 von 12 auf 17 Millionen, im selben Zeitraum wuchs die Zahl der in Argentinien ansässigen jüdischen BewohnerInnen von 218000 auf $378000 .{ }^{20}$ Laut offiziellen Angaben wanderten zwischen

17 Der umliegende Nationalpark in der Region von San Carlos de Bariloche an den Ufern des Sees Nahuel Huapi wurde in den 1950er-Jahren Schauplatz von geheimen Atomexperimenten, welche die argentinische Regierung in Auftrag gab.

18 Die Inbetriebenahme des ersten Skilifts auf dem Cerro Catedral erfolgte 1946. Vgl. Schulz, Bariloche, S. 63.

19 Philipp Mettauer zeichnet in seiner Oral-History-Studie das Schicksal von geflüchteten österreichischen Jüdinnen und Juden nach Argentinien nach. Er geht aber ebenso auf die jüdische Auswanderung aus Europa nach Argentinien vor der NS-Herrschaft ein. Vgl. Mettauer, Erzwungene Emigration, S. 85-86.

20 Vgl. Mettauer, Erzwungene Emigration, S. 85-86. 
1933 und 1943 rund 45000 deutschsprachige Jüdinnen und Juden aus Europa nach Argentinien aus. Sie gründeten dort ebenso eigene jüdische Sport- und Kulturvereine und integrierten sich in bereits bestehende. ${ }^{21}$ Alfredo José Schwarcz schreibt davon, dass jüdische EmigrantInnen bis 1933 großteils in der deutschen Gemeinschaft integriert waren, sich aber mit der Hinwendung der deutschen Vereine zum Nationalsozialismus gezwungen sahen, aus diesen auszutreten. ${ }^{22}$ Sich tatsächlich aus dem Weg zu gehen, funktionierte aber nur bedingt und in Großstädten wie Buenos Aires sicherlich besser als in Kleinstädten der argentinischen Provinz.

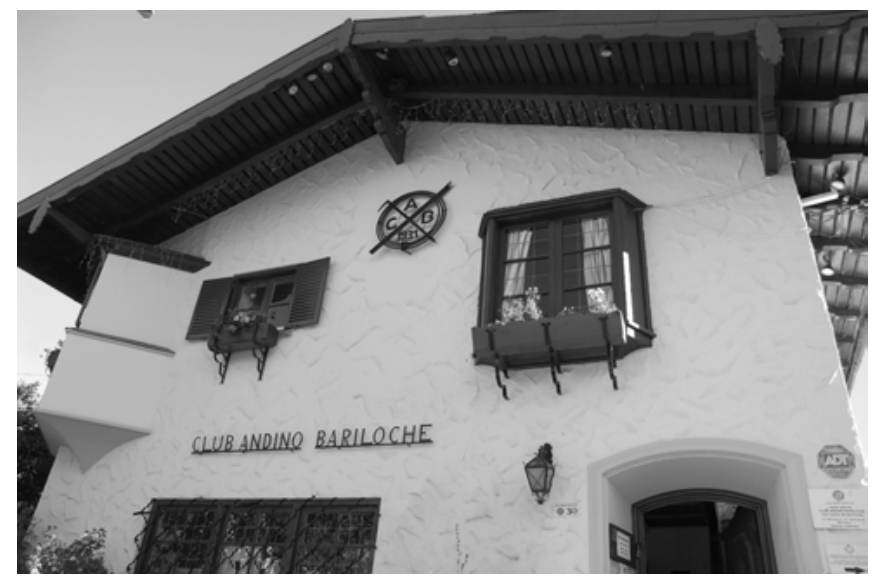

Abb. 38: Der Club Andino Bariloche als „Schmelztiegel“ europäischer EinwandererInnen und Fluchtpunkt belasteter NS-Täter, Praher.

Nach dem Ende des Zweiten Weltkriegs konnten im fernen Argentinien die MitläuferInnen und großteils männlichen Täter des nationalsozialistischen TerrorRegimes untertauchen, ohne sich ihrer Vergangenheit stellen zu müssen. Die ab dem „Anschluss“ 1938 bis 1940 geflüchteten jüdischen Opfer trafen auf ehemalige NS-Verbrecher, im Alltag, auf der Straße und im Vereinsleben. In den argentinischen Anden fuhren sie mitunter gemeinsam Ski, zumindest richteten sie gemeinsame Meisterschaften aus. ${ }^{23}$

21 Vgl. Alfredo José Schwarcz, Trotz allem...Die deutschsprachigen Juden in Argentinien, Wien/Köln/Weimar 1995, S. 60-62 und 155-156.

22 Vgl. Alfredo José Schwarcz, Die deutschsprachigen Juden in Argentinien, in: Holger M. Meding (Hg.), Nationalsozialismus und Argentinien. Beziehungen, Einflüsse und Nachwirkungen, Frankfurt am Main/Berlin/Bern/New York/Paris/Wien 1995, S. 203-226, hier S. 209-210. 23 Die jüdische Auswanderungs- und anschließende Fluchtbewegung der 1930er-Jahre lässt sich in Bariloche nicht nur anhand der Mitgliederverzeichnisse in den Jahrbüchern des Club 
Als in den 1990er-Jahren der NS-Kriegsverbrecher und Massenmörder Erich Priebke im argentinischen Wintersportort Bariloche ausgeforscht und 1995 für den Prozess nach Italien überstellt wurde, führte das international zu einem medialen Echo. Er lebte in der argentinischen Kleinstadt bis zum Auslieferungsverfahren unter seinem richtigen Namen und war wie viele seiner Gesinnungsgenossen ein angesehener Bürger, dessen öffentliches Engagement geschätzt wurde. Priebke betrieb in Bariloche einen Feinkostladen und war Vorsitzender des deutsch-argentinischen Kulturvereins. ${ }^{24}$ Doch nicht nur in diesem Verein fanden ehemalige NS-Funktionäre ein Betätigungsfeld. So existierte in Bariloche seit 1907 eine Deutsche Schule und ab 1931 ein Alpin- und Skiklub und beide Organisationen waren in den 1930er-Jahren nationalsozialistisch unterwandert. $^{25}$

\subsubsection{Die Familie Lantschner und der Club Andino Bariloche}

Der zuvor bereits erwähnte Fritz Lantschner junior, Regierungsdirektor der Abteilung IV der Reichsstatthalterei Tirol-Vorarlberg sowie Vorstandsmitglied der im Krieg gegründeten Skilift AG, ${ }^{26}$ setzte sich vermutlich 1948 ebenso wie sein Bruder Gustav Lantschner nach Argentinien ab. ${ }^{27}$ Die beiden fanden in San Carlos de Bariloche eine Zufluchtsstätte. ${ }^{28}$ Heinz Schneppen nennt den 14. Juli 1948 als Einreisedatum für Fritz Lantschner. Im elektronischen Archivsystem des Museo de la Inmigración in Buenos Aires ist für dieses Datum kein Fritz Lantschner registriert, zumindest taucht unter diesem Namen kein Fritz Lantschner in den Einreiselisten auf. Es besteht daher die Vermutung, dass Lantschner 1948 unter einem Decknamen eingereist sein dürfte. Die Einreise seiner Ehefrau Maria Rosa Lantschner und seiner Kinder von Genua aus in Buenos Aires ist jedenfalls mit 14. Juni 1948 verzeichnet. ${ }^{29}$ Während Gustav Lantschner in dem Anden-Ski-

Andino Bariloche nachvollziehen, sondern auch anhand der von Tabare W. Parsons erstellten Enzyklopädie zur Einwanderungsgeschichte von Bariloche. Vgl. Tabare W. Parsons, Enciclopedia Historica Centenaria de Bariloche 03/05/1902 - 03/05/2002, Almanes y Austriacos Pioneros Olividados, Tomo 1, Bariloche 2002 und Nómina de Socios, in: Anuario Club Andino Bariloche, 18 (1950), San Carlos de Bariloche, 1950, S. 137-152.

24 Vgl. Dieter Wunderlich, Erich Priebke, 2009, https:/www.dieterwunderlich.de/Erich_Priebke.htm (3.7.2019); Klee, Personenlexikon, S. 472-473.

25 Zur Geschichte der Deutschen Schule in Barliloche vgl. Schulz, Bariloche, S. 177.

26 TLA, LG Innsbruck, 10 Vr 924/47.

27 Vgl. Steinacher, Nazis, S. 267

28 Vgl. Schneppen, Odessa, S. 135.

29 Vgl. Certificado De Arribo A América, Maria Rosa Lantschner und Certificado De Arribo A América, Hadwing [sic] Lantschner, Museo de la Inmigración, Centro de Estudios Migratorios 
ort als Skilehrer tätig war, eine Skischule betrieb und Skifilme produzierte, gründete Fritz Lantschner ein Bauunternehmen, das er ab 1961 führte und heute noch seinen Familiennamen trägt. ${ }^{30}$ Die Ermittlungen gegen ihn wegen Beihilfe zum Mord an Franz Hickl beim Juli-Putsch 1934 in Innsbruck gingen ins Leere. Es kam nie zum Prozess. Fritz Lantschner nahm 1957 die argentinische Staatsbürgerschaft an und wurde aufgrund dessen nicht an die österreichischen Behörden ausgeliefert, das Verfahren gegen ihn 1983 eingestellt. ${ }^{31}$ Der Grund, warum sowohl er als auch sein Bruder den Weg nach Bariloche fanden, dürfte der dort schon als Skifunktionär tätige Hans Nöbl gewesen sein. Der in den 1930erJahren ausgewanderte Skisportler und Skilehrer kannte die nun nach Argentinien geflüchteten ehemaligen NS-Skisportler aus seiner aktiven Rennläuferzeit in Tirol. Umgekehrt berichtete die NS-Presse über Nöbls beruflichen Erfolg in den argentinischen Anden. ${ }^{32}$

Der ehemalige Gauamtsleiter Fritz Lantschner wohnte gemeinsam mit seiner Ehefrau Rosa Maria Lantschner, den Kindern und seinem Bruder Gustav unter einem Dach in der Avenida Gallardo und war noch bis in die 1950er-Jahre hinein aktives Mitglied im Club Andino Bariloche. Bei den argentinischen Skimeisterschaften Mitte August 1950 am Cerro Catedral gewann er den Riesentorlauf in der Leistungsklasse der „Veteranen“. Ein Jahr darauf holte Lantschner bei den Senioren erneut den Meistertitel im Riesentorlauf. Seine Tochter Hadwig siegte 1950 bei den Juniorinnen und belegte ebenfalls für den Club Andino Bariloche startend 1951 in der Abfahrt den dritten Platz und wurde im Riesentorlauf Vierte. ${ }^{33}$ Fritz Lantschners Sohn Birger holte 1950 zunächst noch den argentinischen Juniorenmeistertitel im Skilauf, startete aber ein Jahr darauf bereits in der allgemeinen Klasse im Spezialslalom. Dort traf er auf den flüchtigen Jagdflieger Hans-Ulrich Rudel, der ebenfalls als Mitglied im CAB geführt wurde und laut Mitgliedsliste ab 1949 in der Kleinstadt Villa Carlos Paz in der Provinz Cor-

Latinoamericanos (CEMLA), Kopie im Besitz des Verfassers. Der Vorname der Tochter Hadwig Lantschner wurde in den argentinischen Einreisedokumenten fälschlicherweise Hadwing geschrieben.

30 Vgl. Steinacher, Nazis, S. 267; Blaschitz, Austrian National Socialists, o. S., Fußnote 21, https://www.donau-uni.ac.at/dam/jcr:b3e8197f-dd87-4f3e-98be-fc06236dfofe/holocausteraassets_blaschitz.pdf (7.8.2019).

31 Vgl. Schneppen, Odessa, S. 135. TLA, LG Innsbruck, 10 Vr 924/47

32 Hans Nöbl startete zu Beginn der 1930er-Jahre ebenso wie Gustav Lantschner für den Skiklub Innsbruck. Seine Arbeitsmigration nach Argentinien Mitte der 1930er-Jahre war immer wieder Thema in Zeitungsberichten. Vgl. u. a. Tiroler Anzeiger, 22.2.1932, S. 10; Deutscher Telegraf, 31.3.1938, S. 6.

33 Vgl. Anuario Club Andino Bariloche, 19 (1951), San Carlos de Bariloche, 1951, S. 104; Anuario Club Andino Bariloche, 20 (1952), San Carlos de Bariloche, 1952, S. 72-73. 
doba wohnhaft war. ${ }^{34}$ Rudel schrieb zu diesem Zeitpunkt schon für die nationalsozialistisch orientierte Zeitschrift Der Weg (El Sendero), die monatlich in Buenos Aires herausgegeben wurde, und setzte sich unter anderem für die Wiederbewaffnung Deutschlands ein. ${ }^{35}$ Der von der Nachkriegsjustiz gesuchte ehemalige Kampfpilot und Oberst der deutschen Luftwaffe war im Juni 1948 per Flugzeug von Rom nach Buenos Aires geflüchtet. Rudel wurde als einer der Experten im Zuge des argentinischen Raketenentwicklungsprogramms angeworben und als Ausbildner für den militärischen Fliegernachwuchs eingesetzt. ${ }^{36} \mathrm{Er}$ leitete zudem das „Kameradenwerk“, eine Hilfsorganisation, die bemüht war, inhaftierte ehemalige Nationalsozialisten nach Argentinien zu schleusen. ${ }^{37}$

\subsubsection{Treffen mit alten Bekannten aus der „Kampfzeit“}

Im Vereinsjahr 1949 stieß ein anderer alter Bekannter von Fritz Lantschner dazu, der „Alte Kämpfer“ und hochrangige SS-Führer Franz Rubatscher. Er taucht in den Vereinslisten ebenfalls als Mitglied des CAB auf und wohnte ab Anfang der 1950er-Jahre in Bariloche. ${ }^{38}$ Der SS-Obersturmführer Rubatscher war im April 1932 in Innsbruck der NSDAP und SS beigetreten und gehörte der Sicherheitspolizei an. 1939 wechselte er zur Schutzpolizei, wurde aber aufgrund seiner sportlichen Leistungen vom Chef der Sicherheitspolizei, SS-Obergruppenführer Heydrich, zunächst beim SD geführt. Der Bergführer und Skiläufer galt innerhalb der SS und des SD als „hervorragender Sportler“. ${ }^{39}$ Rubatscher holte 1938 den Titel des Deutschen Polizei-Skimeisters und war Inhaber des Reichssportabzeichens in Bronze. Bei der Schutzpolizei wurde Rubatscher, geboren am 19. September 1908 in Innsbruck, mit Wirkung vom 1. April 1943 zum Hauptmann befördert. Er war zu diesem Zeitpunkt in der Dienststelle des SDHauptamtes in München tätig. ${ }^{40}$ Zuvor war Rubatscher im Frühjahr 1941 als Leutnant der Schutzpolizei beim Reserve-Polizei-Bataillon 74 in Krakau einge-

34 Vgl. Anuario Club Andino Bariloche, 20 (1952), San Carlos de Bariloche, 1952, S. 73; Anuario Club Andino Bariloche, 18 (1950), San Carlos de Bariloche, 1950, S. 148.

35 Vgl. u. a. Hans Ulrich Rudel, Wir Soldaten bauen die neue Welt, in: Der Weg (El Sendero), 6 (1950) 4, S. 516-519; Holger M. Meding, „Der Weg“. Eine deutsche Emigrantenzeitschrift in Buenos Aires 1947-1957, Berlin 1997, S. 80-81.

36 Vgl. Meding, „Der Weg“, S. 28.

37 Vgl. Steinacher, Nazis, S. 275.

38 Vgl. Anuario Club Andino Bariloche, 18 (1950), San Carlos de Bariloche, 1950, S. 148.

39 Vgl. Versetzungsverfügung Franz Rubatscher, Berlin 17.5.1944, BArch (ehem. BDC), SSO, Rubatscher, Franz, 19.9.1908.

40 Vgl. Chef der Ordnungspolizei, Oberkommando Polizei, Berlin April 1943, BArch (ehem. BDC), SSO, Rubatscher, Franz, 19.9.1908. 
setzt und dort an der Deportation der jüdischen Bevölkerung beteiligt. ${ }^{41}$ Das brachte ihm im November 1941 die Beförderung zum Oberleutnant ein. ${ }^{42}$ Im Juli 1944 wurde Rubatscher, nachdem er zuletzt weder arbeitsmäßig noch sportlich für den SD tätig gewesen war, seiner Dienststellung als Führer im Reichsicherheitshauptamtes enthoben und zum Führer der Stammabteilung Alpenland in Innsbruck ernannt, die offiziell unter der Bezeichnung SS-Standarte 87 lief. ${ }^{43}$ Rubatscher kehrte damit zu seiner ursprünglichen Wirkungsstätte zurück. Fritz Lantschner und Franz Rubatscher kannten sich seit der Anfangszeit der NSDAP in Innsbruck. Im Zuge der Rückdatierung des Aufnahmetages in die NSDAP gab Lantschner 1937 an, dass sich Rubatscher ,voll und ganz für die Bewegung eingesetzt habe“. ${ }^{44}$ Rubatscher, der nach der Handelsfortbildungsschule eine kaufmännische Lehre absolvierte, war Anfang April 1932 der NSDAP beigetreten und schloss sich zeitgleich der SS-Standarte in Innsbruck an. In seiner achtmonatigen Dienstzeit beim österreichischen Bundesheer absolvierte er zuvor bis Jänner 1930 die Heeresbergführer- und Skilehrerprüfung. Nach dem gemeinsam mit Fritz Lantschner verübten NS-Putschversuch im Juli 1934 wurde er in Haft genommen. Rubatscher saß daraufhin eine mehrmonatige Kerkerstrafe ab und flüchtete ins Deutsche Reich, wo er ab Mai 1936 als Wachtmeister bei der Schutzpolizei München eingesetzt war und nach Besuch der Polizei-Hauptschule in Fürstenfeldbruck 1937 zur Kriminalpolizei Berlin und von dort im August desselben Jahres zur Gestapo München abkommandiert wurde. ${ }^{45}$ Von da an zeigte Rubatschers Karriere steil nach oben. Aufgrund seiner alpinen und skiläuferischen Kenntnisse wurde der SS-Obersturmführer im Frühjahr 1942 als Lehrgangsleiter der Hochgebirgsschule der Ordnungspolizei Innsbruck eingesetzt $^{46}$ und bildete Polizei-Bergführer aus, unter anderem für die in besetzten Gebieten operierenden Polizei-Truppen. ${ }^{47}$ In den Nachkriegsjahren tauschte Ru-

41 Vgl. Krankenblatt Franz Rubatscher, BArch (ehem. BDC), R19/288.

42 BArch (ehem. BDC), SSO, Rubatscher, Franz, 19.9.1908.

43 Vgl. Personalverfügung Franz Rubatscher, Berlin 15. Juli 1944, BArch (ehem. BDC), SSO, Rubatscher, Franz, 19.9.1908.

44 Vgl. Rückdatierung des Aufnahmetages, BArch (ehem. BDC), SSO, Rubatscher, Franz, 19.9.1908.

45 Vgl. Lebenslauf Franz Rubatscher, BArch (ehem. BDC), SSO, Rubatscher, Franz, 19.9.1908; Steinacher, Nazis, S. 274.

46 Vgl. Obersturmführer Franz Rubatscher an den Reichsführer Rasse- und Siedlungshauptamt Berlin, Innsbruck 23.4.1942, BArch (ehem. BDC), SSO, Rubatscher, Franz, 19.9.1908.

47 Zur Hochgebirgsschule der Ordnungspolizei in Innsbruck vgl. u. a. Ralph Klein, Das SSPolizei-Gebirgsjäger-Regiment 18 und seine Bataillone, in: Carsten Gansel/Matthias Braun (Hg.), Es geht um Erwin Strittmacher oder Vom Streit um die Erinnerung, Göttingen 2012, S. 325-362, hier S. 336-337; Ralph Klein, Das SS-Polizei-Gebirgsjäger-Regiment 18, in: Wolfgang Schulte (Hg.), Die Polizei im NS-Staat. Beiträge eines internationalen Symposiums an 
batscher die Alpen gegen die Anden und das argentinische sowie angrenzende chilenische Hochgebirge wurde für ihn und die geflüchteten Lantschner-Brüder Gustav und Fritz zum gemeinsamen Unternehmungsziel. Ende der 1950er-Jahre kehrte Rubatscher gemeinsam mit Gustav Lantschner nach Europa zurück. ${ }^{48}$

Neben Rubatscher trafen die Lantschner-Brüder auf noch einen früheren Tiroler Weggefährten aus Skikreisen, den SS-Hauptsturmführer Hans Aichinger. Die Begegnung war kein Zufall, denn die Lantschner-Brüder hatten ihrem Freund Aichinger bei der Flucht geholfen. ${ }^{49}$ Der staatlich geprüfte Skilehrer, radikale Antisemit und Nationalsozialist Aichinger, dem gemeinsam mit Hubert Salcher im März 1938 die enteignete Skischule von Hannes Schneider zugesprochen wurde ${ }^{50}$ war einer der Haupttäter des Pogroms in Innsbruck im November 1938 und wurde aufgrund dessen von der österreichischen Justiz gesucht. In der Bergwelt von Bariloche fand er eine Zuflucht als Skisportler. Aichinger kehrte 1959 nach Europa zurück und stellte sich den Behörden. Von seiner mittlerweile auf zehn Jahre reduzierten Haftstrafe saß er zweieinhalb Jahre ab und lebte bis 1972 in Innsbruck. ${ }^{51}$

Gustav Lantschner, der ab 1948 in Bariloche ansässig war, hielt sich im Sommer 1953 vorübergehend in Jesolo auf, ehe er am 15. August desselben Jahres laut einem erhalten gebliebenen Brief in Genua das Passagierschiff Castello Verde nach Argentinien besteigen sollte. ${ }^{52}$ Was Lantschner zu diesem Zeitpunkt in Italien machte, geht aus dem Brief nicht hervor. Der Aufenthalt in Europa zeigt aber, dass sich Lantschner in Sicherheit wiegte. Interessant ist in diesem

der Deutschen Hochschule der Polizei in Münster, Frankfurt am Main 2009, S. 201-218, hier S. 203-205.

48 Vgl. Steinacher, Nazis, S. 293.

49 Vgl. Nikolaus Bliem, SS-Hauptsturmführer Johann (Hans) Aichinger, in: Thomas Albrich, Die Täter des Judenporgroms 1938 in Innsbruck, Innsbruck/Wien 2016, S. 58-63, hier S. 62.

50 Vgl. „Exodus österreichischer SkilehrerInnen und die Flucht in den Westen“ in Kapitel 4 der Publikation.

51 Vgl. Bliem, SS-Hauptsturmführer, S. 63.

52 Gustav Lantschner hatte von Robert Matzi aus Argentinien für ein Filmprojekt den Hinweis erhalten, sich ab dem 12.08.1953 in Genua aufzuhalten, um dort das Schiff Castello Verde nach Argentinien zu besteigen. Die anderen Schiffe, Augustus und Caesar seien belegt gewesen, daher habe er die Passage bereits für ihn gebucht. (Brief v. 23.7.53). Zwei Wochen später erhielt Lantschner an seine Adresse in der „Trattoria Roma“ in Jesolo Lido (bei Venedig) einen mit 5. August 1953 datierten Express-Brief der italienischen Reederei „SITMAR“, Società Italiana Trasporti Marittimi. Darin wurde er aufgefordert, sich mit seinem Identitätsausweis im argentinischen Konsulat in Genua am 13. August zwischen 9 und 12 Uhr, den einzigen Öffnungszeiten des Konsulats einzufinden, um seine Ausreisepapiere in Empfang zu nehmen. Nach der Erledigung der Formalitäten ging Lantschner an Bord der Castello Verde und trat am 15. August 1953 die Reise nach Buenos Aires an. Vgl. Nachlass Guzzi Lantschner, WaRis - Tiroler Filmarchiv, Helma Türk \& Dr. Christian Riml. 
Zusammenhang der Absender des Briefes, Robert Matzi. Der gebürtige Wiener war 1949 mit seiner Ehefrau und seinen beiden Kindern nach Bariloche ausgewandert, ${ }^{53}$ ob aus politischen Gründen geflüchtet, konnte nicht geklärt werden. Jedenfalls betrieb der 1918 geborene Matzi im Casa Fitz Roy im Zentrum von Bariloche ein Handelshaus mit regionalen Waren aus heimischer Produktion. ${ }^{54}$ Seine Tochter Catalina Matzi, die das Geschäft der Eltern übernahm, erinnert sich, dass ihr Vater gemeinsam mit Gustav Lantschner Ski- und Bergfilme drehte und sie diese in einem Art Filmstudio gleich neben dem Geschäft produzierten und präsentierten. Ihr Vater sei ebenso wie Gustav Lantschner ein begeisterter Berg- und Skisportler gewesen und hätte sich vor Ausbruch des Zweiten Weltkriegs immer wieder in den Tiroler Bergen rund um Innsbruck aufgehalten. ${ }^{55}$ Die beiden verband demnach eine gemeinsame Leidenschaft.

Gustav Lantschner kehrte Ende der 1950er-Jahre endgültig nach Deutschland zurück und verstarb 2011 bei München. Sein Bruder Fritz blieb in Bariloche. In dem argentinischen Urlaubsort Bariloche wird die Familie Lantschner bis heute geschätzt. Die jüngere Geschichte des Ski- und Alpinklubs ist eng mit jener der ausgewanderten und geflüchteten österreichischen SkisportlerInnen verbunden. Das Bauunternehmen Lantschner ist zudem ein wichtiger Arbeitgeber in der Region. Über die NS-Vergangenheit einzelner Familienmitglieder wurde und wird aber kaum gesprochen. Es ist eine Art „offenes Geheimnis“, schildert der Historiker Hans Schulz, der die Geschichte der deutschen Gemeinde in Bariloche in einem Buch aufgearbeitet hat. ${ }^{56}$ Gemeinsam mit einem Freund und Bekannten bietet er regelmäßig Führungen $\mathrm{zu}$ den Wohnorten und Wirkungsstätten der geflüchteten und international gesuchten ehemaligen Nationalsozialisten und SS-Angehörigen an.

53 Vgl. Parsons, Enciclopedia, S. 191.

54 Vgl. Parsons, Enciclopedia, S. 191.

55 Vgl. Interview mit Catalina Matzi, geführt von Andreas Praher am 17.2.2017 in San Carlos de Bariloche. Laut einer Postkarte, die Leni Riefenstahl am 1.8.1955 aus Mallorca an Gustav Lantschner schrieb, befand sich das Estudio Fitz Roy in der Calle Mitre 58 in Bariloche. Vgl. Postkarte von Leni Riefenstahl an Guzzi Lantschner, Mallorca 1.8.1955, Nachlass Guzzi Lantschner, WaRis - Tiroler Filmarchiv, Helma Türk \& Dr. Christian Riml.

56 Vgl. Gespräch mit Hans Schulz, geführt von Andreas Praher am 19.2.2017 in San Carlos de Bariloche. 


\subsection{Gescheiterte Entnazifizierung}

„Das österreichische Nationalgefühl ging widerstandslos in ein austrofaschistisches, danach in ein nationalsozialistisches und anschließend von einem Tag auf den anderen wieder in ein österreichisches über“57, schreiben Wolfgang Weißgram und Johann Skocek.

Der österreichische Sport spielte sich ebenso schnell und ohne großes Aufsehen von seiner NS-Vergangenheit frei. Der Skisport legte die politische und gesellschaftliche Verantwortung mit der Wehrmachts- und SS-Uniform ab und zog sich stattdessen eine weiße Weste über. „Die Identifikationsangebote sollten in der noch jungen österreichischen Republik in einem hohen Maße von einem funktionierenden rot-weiß-roten Sportbetrieb ausgehen." ${ }^{\text {"58 }}$ Der österreichische Skisport eignete sich hierfür besonders. Medial gepusht und politisch unterstützt, ging der ÖSV dazu über, nationale „SportheldInnen“ zu kreieren. Nicht zuletzt deswegen, weil die Zweite Republik im Zuge des Wiederaufbaus Identifikationsangebote benötigte und nach außen ein positives Image aufbauen wollte. Dem Skisport kam hierbei eine zentrale Rolle zu. ${ }^{59}$ Gleichzeitig wussten es die offizielle österreichische Politik und die Sportpolitik zu nutzen, dass Österreich schon vor seiner Besetzung und Befreiung durch alliierte Truppen auf der Moskauer Konferenz im Oktober 1943 zum ersten „Opfer“ von NSDeutschland erklärt worden ist. ${ }^{60}$ Der Opfermythos wurde dazu verwendet, eine mehrheitsfähige Österreich-Identität zu stiften, ${ }^{61}$ die in den Nachkriegsjahren und -jahrzehnten vielfach vom (alpinen) Skisport genährt wurde. Bevor jedoch der Sportbetrieb der Zweiten Republik aufgebaut werden konnte, mussten die Verantwortung am nationalsozialistischen Terror-Regime von offizieller Seite ausgeschlossen und die Gerichte von der „Unschuld“ der belasteten SportlerInnen überzeugt werden.

57 Skocek/Weisgram, Wunderteam Österreich, S. 202.

58 Praher, Vergessen und verdrängt, S. 358.

59 Der Sporthistoriker Rudolf Müllner spricht in diesem Zusammenhang von einer „Re-Austrifizierung“ durch Sport. Vgl. Rudolf Müllner, Anton Sailer. Österreichs Sportler des Jahrhunderts, in: Matthias Marschik/Georg Spitaler (Hg.), Helden und Idole. Sportstars in Österreich, Innsbruck/Wien 2006, S. 242-258, hier S. 246.

60 Vgl. Robert Knight, Der Waldheim-Kontext: Österreich und der Nationalsozialismus, in: Gerhard Botz/Gerald Sprengnagel (Hg.), Kontroversen um Österreichs Zeitgeschichte, Frankfurt/New York 1994, S. 78-88, hier S. 80-81. Zur Konstruktion des Opfermythos vgl. darüber hinaus Walter Manoschek, Verschmähte Erbschaft. Österreichs Umgang mit dem Nationalsozialismus 1945 bis 1955, in: Reinhard Sieder/Heinz Steinert/Emmerich Tálos (Hg.), Österreich 1945-1995. Gesellschaft - Politik - Kultur, Wien 1995, S. 94-106, hier S. 96-98.

61 Vgl. Oliver Rathkolb, Die paradoxe Republik. Österreich 1945 bis 2005, Wien 2005, S. $47-$ 48. 


\subsubsection{Vom Verbotsgesetz zur Amnestie belasteter SportlerInnen}

Die gesetzliche Grundlage für die juristische Verfolgung ehemaliger Nationalsozialisten bildete das Verbotsgesetz vom 8. Mai 1945. Einen Schwerpunkt darin bildeten die Maßnahmen gegen „Illegale“, also Personen, die während der Verbotszeit der NSDAP vom 1. Juli 1933 bis zum 13. März 1938 der Partei oder einem ihrer Wehrverbände angehört hatten. ${ }^{62}$ Im Verbotsgesetz enthalten waren auch Bestimmungen gegen schwerer belastete NationalsozialistInnen und FördererInnen. ${ }^{63}$ Das waren „politische Leiter“ der NSDAP (vom Ortsgruppenleiter aufwärts) oder Führer von Wehrverbänden (SA, SS). ${ }^{64}$ Ehemalige FunktionärInnen des NS-Reichsbundes für Leibesübungen fielen laut den allgemeinen Bestimmungen in der Verordnung der Bundesregierung vom 10. März 1947 ebenso unter das Verbotsgesetz. ${ }^{65}$ Über die Jahre lockerten sich die Bestimmungen im Rahmen der Entnazifizierung. Im Jahr 1947 begann die Klassifizierung der Ehemaligen in „belastete“ und „minderbelastete“, mit dem Ergebnis, dass von den 537000 registrierten ehemaligen Nationalsozialisten nur noch 42000 „,belastet“ waren. Bereits im Juni 1948 wurden die Sühnefolgen für „Minderbelastete“ aufgehoben und ab 1950 wurden auch „Belastete“ weitgehend amnestiert. ${ }^{66} \mathrm{Im}$ Rahmen des umfassenden Belasteten-Amnestiegesetzes wurden 1952 auch zahlreiche SportlerInnen und SportfunktionärInnen rehabilitiert. ${ }^{67}$ Der Ausschluss belasteter Funktionsträger mündete meist, sofern keine direkte Beteiligung an Kriegsverbrechen nachgewiesen werden konnte, in zeitweiligen Berufsverbo-

62 Beinahe 100000 der rund 550000 im Zuge der Entnazifizierung registrierten NSDAP-Mitglieder galten als „Illegale“. Vgl. Winfried R. Garscha/Claudia Kuretsidis-Haider, Die strafrechtliche Verfolgung nationalsozialistischer Verbrechen - eine Einführung, in: Thomas Albrich/ Winfried R. Garscha/Martin F. Polaschek (Hg.), Holocaust und Kriegsverbrechen vor Gericht. Der Fall Österreich, Innsbruck/Wien/Bozen 2006, S. 11-25, hier S 11.

63 Vgl. StGBl Nr. 13/1945, Verbotsgesetz, Art. III: Bestimmungen gegen „Illegale“, schwerer belastete Nationalsozialisten und Förderer, §10-16.

64 Vgl. Garscha/Kuretsidis-Haider, Die strafrechtliche Verfolgung, S. 11-12.

65 Vgl. Verordnung der Bundesregierung vom 10. März 1947 zur Durchführung des Verbotsgesetzes 1947, StGBl Nr. 64/1947 idF BGBl. Nr. 102/1947, Allgemeine Bestimmungen, § 2.

66 Vgl. Dieter Stiefel, Entnazifizierung in Österreich, Wien 1981, S. 57; Wolfgang Kos, Zur Entnazifizierung der Bürokratie, in: Sebastian Meissl/Klaus-Dieter Mulley/Oliver Rathkolb (Hg.), Verdrängte Schuld, verfehlte Sühne. Entnazifizierung in Österreich 1945-1955, Wien 1986, S. 52-72, hier S. 71.

67 Das umfassende Belasteten-Amnestiegesetz von 1952 beinhaltete auch entsprechende Dienstzeitregelungen. Damit konnten Dienstzeiten während des Nationalsozialismus nachträglich angerechnet werden und Karrieren „repariert“ werden. Vgl. Kos, Zur Entnazifizierung der Bürokratie, S. 71. Die Entnazifizierung der „Belasteten“ endete schließlich mit der endgültigen NS-Amnestie im März 1957, BGBl. Nr.82/1957. 
ten. ${ }^{68}$ Politische Parteien und ihre Interessensverbände hatten hier einen wesentlichen Einfluss auf die Rückkehr der „umworbenen“ SportlerInnen. Das belegen Interventionsschreiben von Dachverbänden im Rahmen von Volksgerichts- und Nachkriegsprozessen. In diesen warben die jeweiligen politisch zugehörigen Verbände (ASKÖ und UNION) um neue Mitglieder. Die Argumentationslinie war dabei stets sehr ähnlich. Sie würden für den Aufbau und die Leitung des nationalen Sportbetriebs gebraucht, hieß es in den Schreiben an die Justiz. Die Beschuldigten selbst beteuerten dagegen ihren „Österreich-Patriotismus“ und betonten in den Vernehmungsprotokollen gleichzeitig ihre sportlichen Leistungen, die sie für Österreich erbracht hätten. Zugleich unterstrichen sie in ihren Aussagen ihre unpolitische Haltung vor und während des Nationalsozialismus und dass ihre Dienstränge in Wehrverbänden ohne ihr Zutun aufgrund ihrer sportlichen Erfolge zustande gekommen waren. ${ }^{69}$

\subsubsection{Der wiedergegründete ösV}

Mit der Wiedereinsetzung der Verfassung am 1. Mai 1945 in der Fassung von 1929 durch die provisorische Staatsregierung erhielt die österreichische Bevölkerung ihre Grundrechte zurück, darunter auch das Recht auf Freiheit der Vereinsbildung. Das Vereins-Reorganisationsgesetz, sprich das Verfassungsgesetz vom 31. Juli 1945, ermöglichte zunächst kommunistischen wie sozialdemokratischen Vereinen die Reaktivierung. Zeitgleich wurden aber auch die Bestimmungen des Stillhaltekommissars für Vereine, Organisationen und Verbände außer Kraft gesetzt. ${ }^{70}$ Für die Vereinsbildung gelangte das Gesetz von 1867 zur Geltung. Die Neubildung eines Vereines musste demnach unter Vorlage der Statuten dem jeweiligen Landeshauptmann angezeigt werden. Aufgrund einer weiteren gesetzlichen Regelung waren die Sicherheitsdirektionen in den jeweiligen Bundesländern zugeschaltet. Wenn ein Verein als „staatsgefährlich“ eingestuft

68 Die Entnazifizierung umfasste im Wesentlichen zwei Bereiche. Die Entnazifizierung nach formalen Kriterien mittels des heute noch in novellierter Form bestehenden Verbotsgesetzes, d.h. Registrierung der NSDAP-Mitglieder, Entziehung ihrer politischen Rechte, Berufsverbote, finanzielle Sühneleistungen etc., und die strafrechtliche Verfolgung von Personen, die nationalsozialistische Verbrechen begangen hatten, nach dem bis 1957 geltenden Kriegsverbrechergesetz. Das Kriegsverbrechergesetz wurde nur von Sondergerichten angewendet, den Volksgerichten. Vgl. Manoschek, Verschmähte Erbschaft, S. 98.

69 Vgl. Praher, Vergessen und verdrängt, S. 359.

70 Vgl. Ulrike Feistmantl, Entnazifizierung und Wiederaufbau des Salzburger Sportwesens, in: Minas Dimitriou/Oskar Dohle/Walter Pfaller/Andreas Praher (Hg.), Salzburgs Sport in der NSZeit. Zwischen Staat und Diktatur, Salzburg 2018, S. 335-356, hier S. 343-345. 
wurde, konnte die Sicherheitsdirektion die Vereinsbildung untersagen. Bei einer länderübergreifenden Vereinsbildung war das Staatsamt für Inneres zuständig. ${ }^{71}$ Hinsichtlich einzelner Vereinsmitglieder orientierte sich das Vereins-Reorganisationsgesetz am Verbotsgesetz.

In einer ersten offiziellen Stellungnahme ließ der wiedergegründete ÖSV nach der Verbandstagung in Kitzbühel im Dezember 1945 über die Medien vermelden, welche SkiläuferInnen nach dem Verbotsgesetz starten dürfen und welche nicht. In der offiziellen Erklärung machte der ÖSV klar, dass „gerade im Skisport die Regelung dieser Frage eine ungemein wichtige und nicht leicht $\mathrm{zu}$ lösende war“. ${ }^{72} \mathrm{Im}$ Vernehmen mit allen Bundesländer-Vertretern legte der ÖSV unter Wahrung der gesetzlichen Bestimmungen fest, dass bei sämtlichen Skiwettbewerben nur Angehörige eines Mitgliedverbandes des ÖSV startberechtigt sind. Die Mitgliedskarte des ÖSV ist dabei vorzuweisen. ${ }^{73}$ Damit sicherte sich der ÖSV als offizieller Skiverband das alleinige Recht, SportlerInnen zu nationalen und internationalen Rennen entsenden zu können. Bereits ein Jahr nach Kriegsende im August 1946 wurde der ÖSV auf dem XVI. Internationalen FIS-Kongress im französischen Pau von 37 versammelten Delegierten aus 18 Ländern als Mitgliedsverband des internationalen Skiverbandes anerkannt und war damit berechtigt, an internationalen FIS-Rennen teilzunehmen. ${ }^{74}$

In der vierten Hauptausschusssitzung des ÖSV, die am 22. und 23. September 1946 in Werfen abgehalten wurde, definierte der Skiverband unter dem Vorsitz von Leopold Spitz seine grundlegenden Ziele. Oberste Priorität hatte laut dem Protokoll der Anschluss an die Spitze im Skilauf, sprich die Förderung des Spitzensports. Um das zu erreichen, waren eigene Ausschüsse für die Schulung der LäuferInnen gebildet worden. Darüber hinaus sollte die Zusammenarbeit mit den Landesverbänden gestärkt werden. Spitz betonte weiter, dass der ÖSV im Gegensatz zu den politisch gebundenen Verbänden, die versuchen würden mit allen Mitteln die Führung an sich zu reißen, nur rein sportliche Interessen

71 Im Jahr 1945 übernahmen die Sicherheitsdirektionen jenen Aufgabenbereich, über den während der NS-Zeit die „Reichsstatthalter“ verfügt hatten. Sie waren dem „Staatsamt für Inneres (Generaldirektion für öffentliche Sicherheit)“ unterstellt und ihrerseits den Bezirksverwaltungsbehörden mit den jeweiligen Polizeibehörden (Polizeidirektionen- und Kommissariate) übergeordnet. Im Falle von länderübergreifenden Vereinsbildungen war nicht mehr die Sicherheitsdirektion, sondern das Staatsamt für Inneres zuständig. Vgl. StGBl Nr. 94/1945, Gesetz vom 20. Juli 1945 über die Überleitung der Verwaltungs- und Justizeinrichtungen des Deutschen Reiches in die Rechtsordnung der Republik Österreich (Behörden-Überleitungsgesetz).

72 Salzburger Volkszeitung, 14.12.1945, S. 3.

73 Vgl. Salzburger Volkszeitung, 14.12.1945, S. 3.

74 Vgl. Protokoll der 4. Hauptausschusssitzung des ÖSV in Werfen, 22./23.9.1946, Mappe ÖSVLänderkonferenz 1946-1948, Archiv Tiroler Skiverband, Kopie im Besitz des Verfassers; FIS, Congress History, 1946-PAU (FRA), https://www.fis-ski.com (6.10.209). 
vertrete. „Unsere Pflicht ist in erster Linie dem österreichischen Skisport zu dienen und nichts weiter zu sein, als nur Sportler!“, stellte Leopold Spitz klar. ${ }^{75}$ Aus den Zeilen des zweiten Vorsitzenden des ÖSV Gotthard Dick geht hervor, dass sich der ÖSV als Verband nicht nur klar von seiner nationalsozialistischen Vergangenheit wie von seiner ausgrenzenden, antisemitischen Politik vor 1938 zu distanzieren versuchte, sondern die politische Verantwortung bei einzelnen Mitglieder verortet sah. 1948 schreibt der ÖSV-Funktionär im offiziellen Jahrbuch Folgendes:

Man griff den Skiverband vor dem Jahre 1938 manchmal auch in der Presse als Naziverband an. Dies mochte wohl bei flüchtiger Betrachtung der Sachlage zutreffen, entsprach aber keineswegs den Tatsachen, weil man übersah, die Sache von der Person zu trennen. Und ich muß ausdrücklich feststellen, daß der Verband damals absolut überpolitisch, die österreichischen Interessen wahrend, geführt wurde. Richtig ist, daß eine Reihe von führenden Funktionären damals dem großdeutschen Gedanken sehr nahe standen. Wie bereits früher erwähnt, hatten im Verbande alle Platz, nach der politischen Einstellung wurde nicht gefragt, man war absolut tolerant. Es herrschte eine ausgezeichnete Skikameradschaft, die nur das eine zum Ziel hatte, Österreichs Weltgeltung im Skilauf zu fördern. Wenn wir uns in die Zeit der politischen Hochspannung zurückversetzen, so werden uns auch verschiedene Handlungen begreiflicher scheinen, was jedoch nicht heißen soll, daß sie auch gebilligt wurden. Es haben einzelne Personen, ob als Wettläufer oder Funktionäre, über das Ziel hinausgeschossen. Es war dies jeweils eine Sache der persönlichen Ansicht des Einzelnen. Aber diese Übergriffe durfte und darf man wohl dem Einzelnen, nicht aber dem Verbande anlasten. ${ }^{76}$

Dick, der dem ÖSV als Hauptausschussmitglied schon von 1928 bis 1938 angehörte, bekräftigte weiter die „sachliche Arbeit“, unterließ es aber zu erwähnen, dass die Toleranzgrenze des ÖSV bei jüdischen Mitgliedern mit der Einführung des „Arierparagraphen“ bereits 1923 erreicht war. Ebenso entsprach seine Sichtweise einer für die Nachkriegszeit üblichen Täter-Opfer-Umkehr, wenn er meinte: „Durch die nationalsozialistische Gesetzgebung war nach 1945 eine Reihe von bewährten Männern zum Zusehen an der Vereinstätigkeit im ÖSV verurteilt.“"77 Dass sich diese „,bewährten Männer“ zum Teil in Wehrverbänden der SA oder SS oder als NSRL-Funktionäre verdient gemacht hatten, blieb in der offiziellen Erzählung unerwähnt. Viel wichtiger war dem wiedergegründeten ÖSV, die Vorrangstellung im österreichischen Skisport wiederzuerlangen:

75 Vgl. Protokoll der 4. Hauptausschusssitzung des ÖSV in Werfen, 22./23.9.1946, Mappe ÖSVLänderkonferenz 1946-1948, Archiv Tiroler Skiverband, Kopie im Besitz des Verfassers.

76 Gotthard Dick, Der ÖSV im Wechsel der Zeiten, in: Skilauf in Österreich. Offizielles Jahrbuch 1948 des Österreichischen Skiverbandes, Wien 1949 S. 21-25, hier S. 24.

77 Dick, Der ÖSV, S. 24-25. 
Der Skiverband soll wieder der große Verband werden, der alle Schichten der Bevölkerung, ohne Unterschied des Standes und der politischen Richtung, erfaßt. Er soll die Brücke bilden, die Trennendes und Vergangenes vergessen läßt, die uns zusammenführt zu einer freudigen Zusammenarbeit im Sport und auch im täglichen Leben. ${ }^{78}$

Die erste wirklich große Skiveranstaltung, die der ÖSV abzuwickeln hatte, war das Glockner-Rennen, das seit 1933 Tradition hatte. Nutzte die nationalsozialistische Sportführung unter tatkräftiger österreichischer Mithilfe im Juni 1938 das traditionelle Skirennen, um den „Anschluss“ und die neue Skimacht „Großdeutschland“ zu propagieren, so sollte das Glockner-Skirennen nach 1945 das neue Österreich präsentieren. Wie wichtig der Politik die Sportveranstaltung war, zeigt sich an der Finanzspritze der Kärntner Landesregierung, die das Rennen mit 3000 Schilling subventionierte. ${ }^{79}$

Der Kärntner Landesverband führte den Mitgliederzuwachs auf über 700 SkiläuferInnen unter anderem auf die erfolgreiche Durchführung der Skisportveranstaltung am Großglockner zurück und hegte Pläne das Skigebiet in Mallnitz lifttechnisch zu erschließen. Mit einem 500 Meter langen Skilift in Mallnitz plante der Kärntner Verband eines der ersten Infrastrukturprojekte zu initiieren. Die Aufstiegshilfe sollte von der Skiliftgesellschaft Bad Gastein finanziert und betrieben werden und der Skilift noch im Winter 1946/47 in Betrieb gehen. ${ }^{80}$

\subsubsection{Der Ruf nach einer Skination}

Der Alpinist, Touristiker und Höhlenforscher Hans Hofmann-Montanus, der ab 1945 auch im Hauptausschuss im ÖSV saß, wollte in seiner Nachkriegs-Fibel Berge einer Jugend, erschienen im Jahr 1948, entsprechend dem Opfernarrativ der Zweiten Republik „alles Politische“ am liebsten zum Teufel jagen. Die individuelle wie kollektive Verantwortung an Nationalsozialismus, Vernichtung und Krieg sollten ausgeblendet werden, die Zweite Republik mit ihren Naturlandschaften einem politikfreien Idyll gleichkommen:

Schriebe der Vielgereiste über Afrika oder Borneo - immer müßt' es zwischen den Zeilen nach Österreich duften wie nach Latschenkiefern. Und - Hand auf die Brust: was wäre

78 Dick, Der ÖSV, S. 25.

79 Leopold Spitz, Protokoll der 4. Hauptausschusssitzung des ÖSV in Werfen, 22./23.9.1946, Mappe ÖSV-Länderkonferenz 1946-1948, Archiv Tiroler Skiverband, S. 2, Kopie im Besitz des Verfassers.

80 Vgl. Protokoll der 4. Hauptausschusssitzung des ÖSV in Werfen, 22./23.9.1946, Mappe ÖSVLänderkonferenz 1946-1948, Archiv Tiroler Skiverband, S. 2, Kopie im Besitz des Verfassers. 
denn, jagt man alles Politische zum Teufel, schöner und näher zum Glück geboren als jenes alte und dieses neuere Österreich? ${ }^{81}$

Der Leiter des Salzburger Landesverkehrsamtes in der Ersten Republik und im Austrofaschismus war in den 1920er-Jahren unweigerlich mit der ausgrenzenden Politik des ÖSV in Berührung gekommen. Hofmann-Montanus war Mitbegründer des niederösterreichischen Landesskiverbandes und ÖSV-Funktionär nach dem Ersten Weltkrieg. 1926 wurde der Touristiker von Wien nach Salzburg berufen. Zunächst in der Ersten Republik und danach im Austrofaschismus hatte er in seinem Amt als Leiter des Salzburger Landesverkehrsamtes weitreichende politische Kompetenzen und entschied in Abstimmung mit dem Salzburger Landeshauptmann Franz Rehrl über Förderzusagen für den Ausbau der touristischen und sportlichen Infrastruktur. 1938 endete dieser Einfluss. Als austrofaschistischer Landesbeamter und Mitglied der Vaterländischen Front musste er sein Amt räumen. Die Nationalsozialisten suspendierten Hofmann-Montanus am 16. März 1938 von seinem Landesdienst und nahmen ihn drei Tage in Gewahrsam. Ende Mai 1938 wurde er erneut in Haft genommen und zuvor seine Wohnung durchsucht. Einen Monat später kam er wieder frei und wurde sogleich in den Ruhestand versetzt. ${ }^{82}$ Im Dezember 1939 wurde er wegen Veruntreuung und Missbrauch der Amtsgewalt am Landesgericht Salzburg angeklagt und $\mathrm{zu}$ zwei Jahren schwerer Kerkerhaft verurteilt. Hofmann-Montanus hatte laut Urteil zwischen 1930 und 1938 an die 7000 Schilling an Spesenpauschalen veruntreut. Die Strafe galt aber bereits im Jänner 1940 als getilgt. Nach 1945 deklarierte er sich als Opfer der NS-Herrschaft, er hätte „schwere Verfolgungen“ zu erdulden gehabt. Er sei zudem für einige Wochen Zellengenosse des Salzburger Landeshauptmannes Franz Rehrl gewesen. ${ }^{83}$ Fakt ist, Hofmann-Montanus machte nach 1945 wiederum Karriere in der Salzburger Landesregierung und unterstützte als abermaliger Leiter des Landesverkehrsamtes wie schon vor 1938 den regionalen Sportbetrieb immer im Hinblick auf den touristischen Nutzen. ${ }^{84}$ Hofmann-Montanus wurde mit Wirkung vom 1. Mai 1945 am 20. August 1949 in den Landesdienst aufgenommen. Er durchlebte in seiner beruflichen Laufbahn, die durch den Nationalsozialismus unterbrochen wurde, mehrere Umbrüche und Systemwechsel, verhielt sich dabei stets politisch unauffällig und passte sich den jeweiligen Gegebenheiten mehr oder minder erfolgreich an.

81 Klappentext zu Hans Hofmann-Montanus, Berge einer Jugend, Wien 1948.

82 Vgl. SLA, Bestand Personalakten Landesregierung, Personalakt 1801/1, Hans HofmannMontanus; NS-SOKO A 64.031.

83 Vgl. SLA, Bestand Personalakten Landesregierung, Personalakt 1801/1 und Personalakt 1801/2, Hans Hofmann-Montanus.

84 Vgl. Zeisberger/Heinisch (Hg.), Leben über den Tod, S. 366. 
Als hochdekorierter Erster Weltkriegsteilnehmer, der unter anderem am Ortler für die Skitruppen gekämpft hatte, ${ }^{85}$ war er einer jener Vertreter, denen die „Bergheimat“ bis zum Schluss ein nationales Anliegen war und für die er sich auch nach 1945 einsetzte. Die Sichtweise von Hofmann-Montanus teilten auch andere. So schrieb der Alpin- und Skipublizist Günther Flaig 1947:

\begin{abstract}
Der riesige Zuschauerstrom, der die glitzernden Kampfbahnen der großen Skirennen und Meisterschaften umflutet, beweist das außerordentliche Interesse des ganzen Volkes an diesem herrlichsten aller Sporte. Vom Ministerbüro und Großhotel bis zur kleinsten Werkstatt und letzten Bergbauernhütte - überall wird über die Läufer und Skirennen gesprochen, gestritten, gewettet. [...] Wir haben genügend Abstand von den zwei Nachkriegswintern 1945/46, 1946/47, um die Entwicklung zu beurteilen, die der österreichische Skisport in diesen entscheidenden Jahren des Wideraufbaues durchgemacht hat. Auf alpinem Gebiet, also in Abfahrt und Torlauf, war Österreich noch niemals auf dieser hohen Stufe wie heute, wenn dies auch - wenigstens bei den Herren - noch nicht durch erste Ränge in internationalen Wettkämpfen zum Ausdruck kam. Der Gradmesser der Öffentlichkeit für den Leistungsstand der Skinationen sind die Siegerlisten der größten Rennen jedes Winters. $^{86}$
\end{abstract}

\title{
6.2.4 Erste skisportliche Aktivitäten in Salzburg und das Buhlen um belastete Sportler
}

Während sich beim oberösterreichischen Landesskiverband die behördliche Genehmigung zu spießen schien, aber dann mit Herbst 1946 doch auf den Weg gebracht werden konnte, ${ }^{87}$ hatte der Skiclub Salzburg als Mitgliedsverein des Landesskiverbandes sein Bestreben einer Neugründung bereits im März 1946 durchgesetzt. In den eingebrachten Vereinssatzungen wurden ehemalige Angehörige der SS grundlegend abgelehnt und Angehörige der SA nur als Mitglieder akzeptiert, sofern sie keinen Dienstrang bekleidet hatten. ${ }^{88}$ Während dieser Passus bei der Fassung der Statuten 1946 unter Mitgliedschaft noch explizit erwähnt wurde, fehlte er 1952 komplett. In den nun neu formulierten Satzungen des SCS konnte plötzlich jede Person, die das 18. Lebensjahr vollendet hatte, ordentliches Mitglied werden. ${ }^{89}$ Die Kriterien, um als Vereinsmitglied aufge-

85 Vgl. Lebenslauf Hans Hofmann-Montanus, SLA, Bestand Personalakten Landesregierung, Personalakt 1801/1.

86 Flaig, Skikanonen, o. S.

87 Vgl. Flaig, Skikanonen, S. 3

88 Vgl. SLA, Sicherheitsdirektion (SID), aufgelöste Vereine 1946, 657/47 Skiclub Salzburg (SCS), Vereinsbildung.

89 Vgl. SLA, Sicherheitsdirektion (SID), aufgelöste Vereine 1946, 657/47 Skiclub Salzburg (SCS), Vereinsbildung. 
nommen zu werden, unterlagen nach der ersten Amnestiewelle keinen so strengen Regeln mehr. Die Öffnung des Vereins nach außen und die Initiative des Skiclubs Salzburg jetzt auch verstärkt vormals belastete SportlerInnen als Mitglieder anzuwerben, war eine logische Konsequenz und funktionierte. Manche befanden sich auch schon dort, obwohl sie eigentlich laut Statut von 1946 nicht dort sein hätten dürfen. So startete der bereits in der Zwischenkriegszeit erfolgreiche Harald Bosio ab Jänner 1946 für den Salzburger Skiclub. ${ }^{90}$ Der staatlich geprüfte Skilehrer aus Judenburg war 1934 in die NSDAP eingetreten und ab 1936 SA-Mann und als solcher Sportreferent in der SA-Standarte 4/84. ${ }^{91}$ Beim SCS war Bosio in der Nachkriegszeit nicht nur sportlich aktiv, sondern ab Oktober 1952 auch als Beisitzer im Arbeitsausschuss tätig. ${ }^{92}$

Der Skiclub Salzburg wurde mit seiner aktiven Mitgliederpolitik zu einem Sammelbecken ehemaliger SpitzensportlerInnen des Deutschen Reichs, die ihre politische Vergangenheit dort verschleiern konnten. Auf diese Weise fand auch der Sudetendeutsche Heinrich (Heinz) Palme, der im Dezember 1941 als Skispringer für die Ordnungspolizei Innsbruck startete, zum SCS. ${ }^{93}$ Der Skispringer aus dem Riesengebirge war ebenso wie Josef Bradl Mitglied der reichsdeutschen Ski-Nationalmannschaft. ${ }^{94}$ Palme nahm Ende Jänner 1941 neben Bradl und dem aus Thüringen stammenden SS-Skisportler Rudi Gehring an den Deutschen Skimeisterschaften in Spindelmühle teil. Er startete damals als Angehöriger der Staatspolizeistelle Innsbruck für die Skimannschaft der Polizei Innsbruck. ${ }^{95}$ Drei Jahre später, im März 1944, trafen Bradl, Gehring und Palme bei einem Skisprungbewerb im besetzten Zakopane unweit des KZ-Außenlagers Olcza erneut aufeinander. ${ }^{96}$ Schon kurz nach der Wiedergründung und behördlichen Zulas-

90 Salzburger Nachrichten, 22.1.1946, S. 4.

91 Vgl. BArch (ehem. BDC), PK, Bosio, Harald, 02.01.1906.

92 Vgl. SLA, SID, aufgelöste Vereine 1946, 657/47 Skiclub Salzburg (SCS), Vereinsbildung.

93 Der Skispringer Heinrich (Heinz) Palme, geboren am 20.5.1912 in Gablonz (Böhmen), wird in Zeitungsberichten und in der Sekundärliteratur mit abwechselnden Vornamen erwähnt. Vgl. Kleine Volks-Zeitung, 29.12.1941, S. 5; Gerd Falkner/Klaus-Dieter Blühm, Zeitreise. Auf Skiern durch Sachsen. 100 Jahre Skiverband Sachsen, Aachen 2008. Ebenso diente im Konzentrationslager Dachau ein SS-Unterscharführer mit dem Namen Heinrich Palme. Ob dieser mit dem Skispringer Heinrich Palme identisch ist, konnten erste Recherchen nicht klären. Vgl. Benz/ Distel (Hg.), Der Ort des Terrors, 2005, S. 306.

94 Vgl. u. a. Innsrbucker Nachrichten, 31.1.1941, S. 12.

95 Vgl. Kleine Volks-Zeitung, 27.1.1941, S. 4.

96 Vgl. Innsbrucker Nachrichten, 6.3.1944, S. 4; Wolfgang Benz/Barbara Distel (Hg.), Der Ort des Terrors. Geschichte der nationalsozialistischen Konzentrationslager. Riga-Kaiserwald, Warschau, Vaivara, Kauen (Kaunas), Plaszów, Kulmhof (Chelmo), Belzec, Sobibór, Treblinka (Band 8), München 2008, S. 298. 
sung des Skiklubs Salzburg im Dezember 1946 startete Palme für den SCS. 1952 übernahm der ehemalige erfolgreiche NS-Skispringer als erster Sportwart die sportliche Leitung im Verein. ${ }^{97}$

\subsubsection{Die Etablierung der Vereinsarbeit}

Innerhalb weniger Jahre nach den Wiederzulassungen und Neugründungen hatte sich die Vereinsarbeit des ÖSV zu Beginn der 1950er-Jahre etabliert. Sie hatte einen wesentlichen Anteil daran, dass die junge sportreibende Generation im Skibetrieb der Zweiten Republik Fuß fassen konnte. In welchem Ausmaß das geschah, verdeutlichen die Mitgliederzahlen der Landesverbände im ÖSV für die Saison 1952/53. Insgesamt waren im ÖSV mit 30. Juni 1953 über 32600 Mitglieder registriert, die meisten von ihnen in den Landesverbänden Salzburg, Tirol, Kärnten und Osttirol sowie Vorarlberg. Die Zahlen geben Aufschluss darüber, wie schnell sich der vereinsmäßig organisierte österreichische Skisport von den Folgen des Zweiten Weltkriegs erholen konnte, zu einem Zeitpunkt in dem die schwache Nachkriegswirtschaft und hohe Arbeitslosigkeit noch zu einer Abwanderung von Arbeitskräften führten. ${ }^{98}$ Einer Wachstumsrate von zwölf Prozent stand in den Jahren 1946 bis 1952 eine Inflationsrate von 37 Prozent gegenüber. $^{99}$

97 Vgl. SLA, SID, aufgelöste Vereine 1946, 657/47 Skiclub Salzburg (SCS), Vereinsbildung; Salzburger Volkszeitung, 7.1.1947, S. 4.

98 Österreich war in den Nachkriegsjahren ein Auswanderungsland. Neben Flüchtlingen und DPs, die nach dem Ende des Zweiten Weltkriegs in Österreich strandeten und meist weiter auswanderten, suchten tausende junge österreichische Frauen und Männer in den 1950erund 1960er-Jahren aufgrund des langsamen Wirtschaftswachstums und der hohen Arbeitslosigkeit eine neue Perspektive in der Arbeitsmigration. Vgl. u. a. Hahn, Historische Migrationsforschung, S. 179-180 und 188.

99 Vgl. Ernst Hanisch, Der lange Schatten des Staates. Österreichische Gesellschaftsgeschichte im 20. Jahrhundert, Wien 1994, S. 438. 


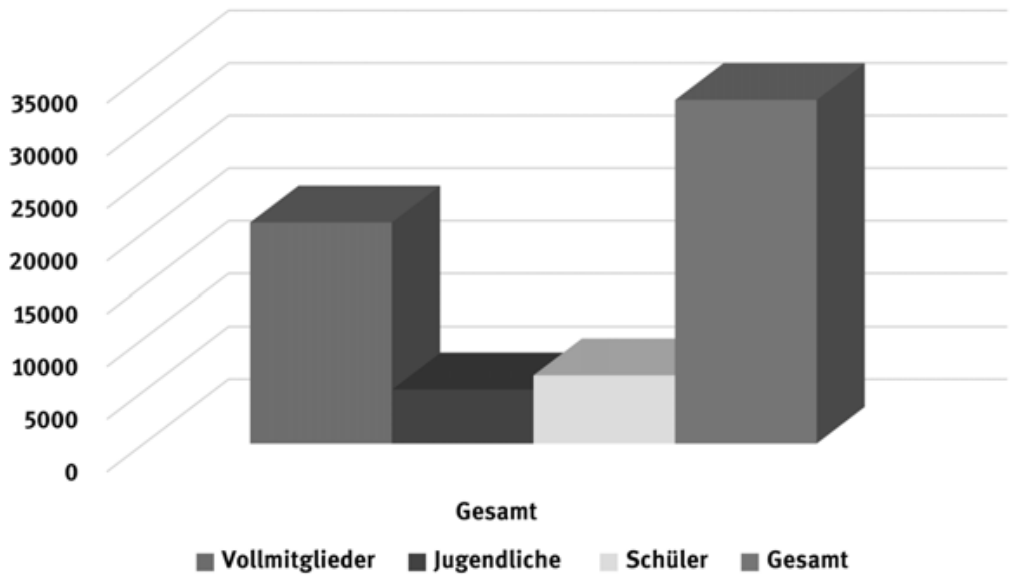

Grafik 8: Mitgliederstand im ÖSV gesamt, Stand Juni 1953.

Quelle: Ski Sport 2 (1953/54) 1, S. 6, eigene Zusammenstellung.

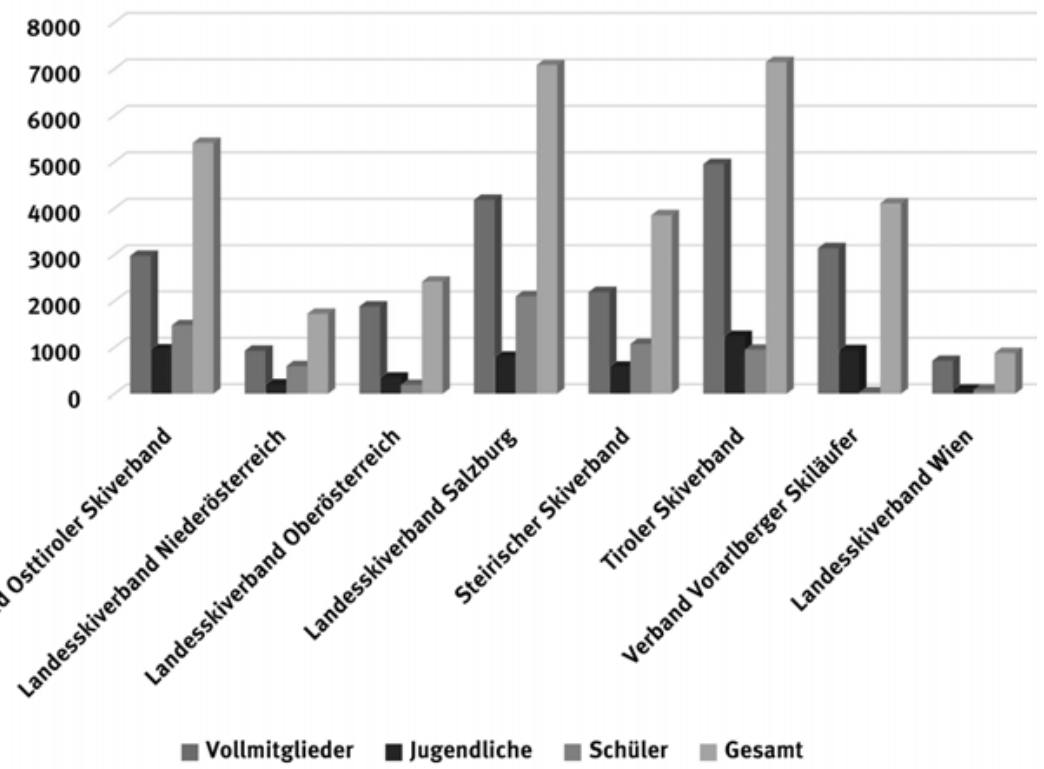

Grafik 9: Mitgliederstand im ÖSV nach Landesverbänden, Stand Juni 1953. Quelle: Ski Sport 2 (1953/54) 1, S. 6, eigene Zusammenstellung. 


\subsection{Erste internationale Starts und der starke weibliche Skinachwuchs}

Die ersten Erfolge im österreichischen Nachkriegsskisport schrieben aber mehr die Frauen als die Männer. Diese galten in der Regel eher als unbelastet als die in Lagern internierten bzw. in Nachkriegsprozessen angeklagten ehemaligen SA- und SS-Sportler. In der Wintersaison 1946/47 waren österreichische SkisportlerInnen erstmals nach dem Zweiten Weltkrieg wieder für internationale Rennen im Ausland zugelassen. $\mathrm{Zu}$ den ersten großen Skiveranstaltungen mit österreichischer Beteiligung zählten das Weiße Band in St. Moritz und das Kandahar-Rennen in Mürren. ${ }^{100}$ Beide Wettbewerbe fanden auf neutralem Boden in der Schweiz statt. Für die Sensation bei den Frauen sorgte die 22-jährige Bankangestellte aus dem Kleinwalsertal Resi Hammerer. Die gebürtige Vorarlbergerin, Jahrgang 1925, siegte in St. Moritz in der Abfahrt und Kombination. Im selben Jahr holte sie im Kombinations- und Spezialtorlauf den österreichischen Meistertitel und wurde in der Folge für den Olympiakader nominiert. ${ }^{101}$ In ihrer Jugend startete Hammerer für das Deutsche Reich und konnte bei den Deutschen Skimeisterschaften in den alpinen Bewerben am Arlberg im März 1944 in der allgemeinen Spitzenklasse sowohl im Torlauf als auch in der Kombination den dritten Platz belegen. Im Torlauf wurde Hammerer allerdings ein paar Tage später zugunsten ihrer Alterskonkurrentin Annelore Zückert strafverifiziert und auf den vierten Rang zurückgereiht. ${ }^{102}$ Dennoch zählte sie gegen Ende des Zweiten Weltkriegs zum jungen Favoritinnen-Kreis im reichsdeutschen Skilauf. Ihre Skikarriere wurde, wie die anderer ihrer Generation, maßgeblich von Christl Cranz beeinflusst, die als Spitzensportlerin, NS-Ski-Idol und BDM-Führerin prägend war. ${ }^{103}$ Ebenso wie andere ihres Jahrgangs durchlief Hammerer die ideologische und sportliche Kaderschule des BDM. Nun stand die junge ÖSV-Sportlerin mit der Aussicht auf die Olympia-Teilnahme kurz vor dem Höhepunkt ihrer sportlichen Karriere. Bei den Olympischen Winterspielen 1948 in St. Moritz holte sie schließlich Bronze für Österreich. ${ }^{104}$

Eine andere Nachwuchshoffnung des österreichischen Skisports der unmittelbaren Nachkriegszeit war Annelore Zückert. Die aus Grödig stammende Skirennläuferin wurde im selben Jahr wie Hammerer geboren, sie kam am 5. Febru-

100 Flaig, Skikanonen, o. S.

101 Vgl. Flaig, Skikanon, o. S.; Ilse Korotin (Hg.), biografiA. Lexikon österreichischer Frauen, Band 1, A-H, Wien/Köln/Weimar 2016, S. 1172.

102 Vgl. Neues Wiener Tagblatt, 6.3.1944, S. 4; Innsbrucker Nachrichten, 14.3.1944, S. 4.

103 Vgl. Hofmann, Christl Cranz, S. 1013-1029.

104 Vgl. Korotin, biografiA, S. 1172. 
ar 1925 zur Welt. Zückert duellierte sich seit ihrer Jugend mit Resi Hammerer und arbeitete sich von der BDM-Skiläuferin zur Gaumeisterin von Salzburg hoch. ${ }^{105}$ Der nachträglich an Zückert vergebene dritte Platz bei den Deutschen Skimeisterschaften bedeutete den größten Erfolg für die vom BDM aufgerückte NSRL-Skisportlerin. Im Februar 1946 entschied sie in Abfahrt, Slalom und Kombination die erste Salzburger Landesmeisterschaft für sich, damals für den Skiclub Salzburg, ${ }^{106}$ und wurde 1947 österreichische Meisterin vor Dagmar Rom und Resi Hammerer. Aufgrund ihrer Erfolge, Hahnenkammsiegerin in Kitzbühel und Goldener Ring von Seefeld, wurde Zückert 1947 ebenso wie Hammerer in den Olympiakader berufen. Sie erreichte bei den Olympischen Winterspielen 1948 in St. Moritz nur den 16. Platz. Dafür gewann sie im selben Jahr den Riesentorlauf in Grindelwald. Nach ihrer aktiven Rennkariere arbeitete die ausgebildete Heilgymnastin als Trainerin des Skiclubs Salzburg und Amateurtrainerin der jugoslawischen Damen-Nationalmannschaft. ${ }^{107}$

Zum Star des österreichischen Nachkriegsskisports entwickelte sich die bereits erwähnte Dagmar Rom. Nachdem sie vom Unterrichtsministerium zum Studium zugelassen wurde, inskribierte sie zunächst 1946 an der Universität Innsbruck Sport und Geografie und feierte im Jänner 1947 bei den Akademischen Weltmeisterschaften im Schweizer Davos mit drei Goldmedaillen ihren ersten großen internationalen Erfolg. Das war gleichzeitig ihr Ticket für die österreichische Nationalmannschaft und bedeutete die Qualifikation für die Olympischen Spiele in St. Moritz. Dort erlitt Rom im Training eine Knieverletzung und konnte nicht starten. Dafür krönte sie sich eine Wintersaison später in Aspen/Colorado in Slalom und Riesentorlauf zur Doppelweltmeisterin. ${ }^{108}$ Gleichzeitig fand Rom zum Skifilm und wurde durch diesen bekannt. Sie bekam das Angebot, in Nacht am Mont Blanc (1951) die Hauptrolle zu spielen. Das Drehbuch schrieb der langjährige Riefenstahl-Wegbegleiter Harald Reinl, der auch Regie führte. Rom erinnert sich: „Damals gab’s nur das, nur dieses Skifahren. Da war noch kein Tennis, noch keine Leichtathletik. Da haben sie sich um mich gerissen überall. [...] Wir haben zehn Filme anfangen wollen, nur Weiße Hölle am Mont Blanc ist fertig geworden. “109 Rom zierte daraufhin die Titelblätter von in- und ausländischen Magazinen. Ende 1950 wurde sie in Österreich zur

105 Vgl. Völkischer Beobachter, 15.3.1944, S. 4.

106 Vgl. Salzburger Volkszeitung, 4.2.1946, S. 3.

107 Vgl. Praher, Politisch belastet, S. 370; Flaig, Skikanonen, o. S.; 100 Jahre Skiclub Salzburg, S. 54; Hubert Becker, Ski-Club Grödig. Rückblick, Einblick, Ausblick, Salzburg 1987, S. 43; Marktgemeinde Grödig (Hg.), Festschrift zur Markterhebung von Grödig, Salzburg 1968, S. 85.

108 Vgl. Interview mit Dagmar Rom; Gidl/Graf, Skisport, S. 109.

109 Interview mit Dagmar Rom. 
„Sportlerin des Jahres“ gewählt. Sie war der erste von den Medien gefeierte weibliche Sportstar der Zweiten Republik. Sie wurde als Ehrengast zur Eröffnung des ersten Radrennens auf der Krieau eingeladen und zu einem Vortrag vor 900 ZuhörerInnen an die Universität Wien. ${ }^{110}$ Der Gegensatz zum ersten Auslandstart in Davos im Jänner 1947 hätte nicht krasser sein können: „Wir haben kaum was zu essen gehabt. Unser Proviant waren gekochte Kartoffel und ein Teesackerl. Das haben wir mitgehabt und auf der Hütte Wasser bestellt.“111

Trotz dieser Erfolge schrieb Günther Flaig von einer „Ernüchterung“, da die Männer in der Wintersaison 1946/47 im Unterschied zu den Frauen in den Siegerlisten weniger häufig vertreten waren. ${ }^{112}$ Gegen Anfang der 1950er-Jahre sollte sich dieses Bild zugunsten der aus Internierungslagern freigelassenen und in Entnazifizierungsprozessen freigesprochenen männlichen Spitzensportler ändern. Sie kehrten wieder auf die Bühne des Sportgeschehens zurück und eroberten diese für sich.

\title{
6.4 Die Olympischen Winterspiele in St. Moritz
}

Die Olympischen Winterspiele in St. Moritz 1948 wurden zur ersten Bewährungsprobe des österreichischen Skisports nach dem Zweiten Weltkrieg auf internationaler Bühne.

\begin{abstract}
Zum 1. Male nach dem unglückseligen Krieg nahm Österreich wieder als gleichberechtigter Partner an einer Weltkonkurrenz teil. Unter den 31 Völkern, die in St. Moritz versammelt waren, gab Österreich sein Bestes, im Interesse des Sportes und zur Wahrung des Ansehens Österreichs. Österreich hat es bald nach der Befreiung verstanden, den Anschluß an die Welt wieder zu gewinnen. Es ist heute auf dem Gebiet des Sportes Mitglied aller internationalen Sportorganisationen und damit berechtigt, auch an der größten Sportdemonstration der Neuzeit, der Olympiade teilzunehmen ${ }^{113}$
\end{abstract}

schrieb Josef Gerö, der Präsident des Österreichischen Olympischen Comités (ÖOC) in einem Vorwort zu der Olympia-Sonderausgabe der Wiener Illustrierten 1948.

110 Vgl. Gidl/Graf, Skisport, S. 109.

111 Interview mit Dagmar Rom.

112 Flaig, Skikanonen, o. S.

113 Dr. Gerö, Präsident des Olympischen Komitees, in: Wiener Illustrierte, Sonderaugabe zur Winter-Olympiade St. Moritz 1948. Josef Gerö war Jurist jüdischer Herkunft und bis 1938 Präsident des Wiener Fußballverbandes. Während der Zeit des Nationalsozialismus befand er sich in KZ- und Gestapo-Haft. Nach 1945 war er Präsident des ÖFB und Justizminister bis 1949 bzw. von 1952-1954. Vgl. u. a. John, Donaufußball, S. 208. 
Die österreichische Olympia-Delegation musste zwar auf ihren Skisprungstar Josef Bradl verzichten, konnte aber dafür auf andere männliche Spitzensportler zurückgreifen, die im nationalsozialistischen Sportsystem aufgestiegen waren und während der nationalsozialistischen Herrschaft nicht nur sportlich aufgefallen waren. Der 1915 geborene nordische Kombinierer Paul Haslwanter war einer von ihnen.

Den Erhebungen im Rahmen des Entnazifizierungsprozesses zufolge war der in Seefeld gebürtige Landwirt und staatlich geprüfte Skilehrer Paul Haslwanter seit 1933 Mitglied der NSDAP und seit 1. März 1935 Angehöriger der SS. Genau gesagt, gehörte er dem SS-Sturm in Seefeld an und war ab 1938 SS-Rottenführer im Sturm 3/87. ${ }^{114}$ Aufgrund dessen wurde er 1946 gemäß dem Verbotsgesetz für die Dauer von sechs Monaten zu Wiederaufbauarbeiten herangezogen ${ }^{115}$ und war zum Zeitpunkt der Vorbereitungen auf die Olympischen Spiele in St. Moritz 1948 in der Gemeinde Seefeld sowie in der Landwirtschaft seines Vaters tätig. Schon 1946 startete Haslwanter bei seinen ersten Rennen und war durchaus erfolgreich. So holte er bei der ersten Tiroler Meisterschaft nach dem Zweiten Weltkrieg in seiner Heimatgemeinde Seefeld im Jänner 1946 den Kombinationssieg und machte auf sich aufmerksam. „Tirol hat als erstes Bundesland im befreiten Österreich seine Skimeister im Langlauf und Springen in Seefeld ermittelt“, ${ }^{116}$ schrieb das Salzburger Tagblatt. Haslwanter konnte sich in Folge mit einem zweiten Platz in der Kombination und einem dritten Platz im Langlauf bei den österreichischen Meisterschaften empfehlen. ${ }^{117}$ Damit schaffte er den Sprung in den Olympia-Kader. Im Jänner 1947 befand sich Haslwanter mit anderen Rennläufern auf dem Olympialager in Kitzbühel. Während der Vorbereitungen mussten die ausgewählten Olympiastarter den Reisepassantrag für die Einreise in die Schweiz ausfüllen. Da mit seinen wahrheitsgetreuen Angaben eine Einreise unmöglich schien, hatte ihm der Leiter des Olympialagers Sepp Kerscher geraten, er solle seine Zugehörigkeit zur NSDAP nicht erwähnen. ${ }^{118}$ Haslwanter befolgte den Rat und konnte trotz seiner SS-Vergangenheit in St. Moritz starten. „Die Olympiamannschaft Österreichs marschiert ein! Stolz trägt Haslwanter das Schild ,Autriche““, schrieb die Wiener Illustrierte in ihrer

114 Vgl. TLA, LG Innsbruck, Vr 1720/46; BH Innsbruck, Entnazifizierung, Karton 724/28, Akt 2554/48; Sonderbehörden nach 1868 - Sicherheitsdirektion für Tirol (ATLR Abteilung VII) 1946 - Pos. 5119.

115 Vgl. BH Innsbruck, 15.5.1946 an Paul Haslwanter, TLA, BH Innsbruck, Entnazifizierung, Karton $724 / 28$, Akt 2554/48.

116 Salzburger Tagblatt, 31.1.1946, S. 7.

117 Vgl. Flaig, Skikanonen, o. S.

118 Vgl. Niederschrift, Gend. Postenkommando Seefeld, 8.11.1948, aufgenommen mit Paul Haslwanter, BH Innsbruck, Entnazifizierung, Karton 724/28, Akt 2554/48. 
Sonderausgabe unter das Bild vom Einzug der österreichischen Olympia-Delegation. ${ }^{119}$

Für einen Stockerlplatz reichte es nicht, dafür holte Trude Beiser Gold in der alpinen Kombination und damit Österreichs einzige Medaille. Für Haslwanter waren die Spiele in der Schweiz dennoch ein persönlicher Erfolg, er galt mit seiner Teilnahme als rehabilitiert. Der Gendarmerieposten Seefeld stellte ihm nach einer nochmaligen staatsrechtlichen Überprüfung einen guten Leumund aus und schrieb im November 1948 an die Staatsanwaltschaft, Haslwanter habe „einen guten Ruf, ist allseits beliebt und als begeisterter Sportler Schifahrer [sic] bekannt“. ${ }^{120}$

\subsection{Das Opfernarrativ und der Beitrag der Familie Flaig für den österreichischen Nachkriegsskisport}

Dass sich der österreichische Skisport als Opfer stilisierte, belegt die Darstellung von Günther Flaig in seiner Skifibel. Darin schreibt der Alpin- und Skikolumnist: „Während des Krieges hatten die Schweiz, Frankreich und Italien gegenüber Österreich entscheidende Vorteile, konnten doch deren Mannschaften zum Großteil im Training bleiben und hatten fast keine Ausfälle.“121

Mit Hilfe dieser These begründet Flaig das schlechte Abschneiden der österreichischen SkisportlerInnen bei den Skirennen in der Saison 1946/47. Er verkennt damit die Tatsache, dass viele, vor allem männliche Spitzenläufer, in SSoder Polizeieinheiten sehr wohl im Training standen und auch Sportlerinnen, die über den BDM eine sportliche Schulung genossen, zur Ausübung des Skilaufs gelangten ebenso wie ihre jungen männlichen Kollegen bei der HJ. Außerdem lässt er außer Acht, dass Frankreich von deutschen Truppen besetzt war und sich italienische Skisportler ebenso wie österreichische oder reichsdeutsche im Kriegseinsatz befanden. Von einem Wettbewerbsvorteil aufgrund eines Trainingsvorsprungs konnte also keine Rede sein. Diese historische Missinterpretation muss wohl auch im Kontext seiner Biografie und seines familiären Hintergrundes gesehen werden. Günther Flaig war der Sohn des bereits erwähnten Nationalsozialisten Walther Flaig, der bereits vor 1938 öffentlich für die nationalsozialistische Bewegung auftrat und seinen Sohn bei Bergtouren regelmä-

119 Wiener Illustrierte, Sonderausgabe zur Winter-Olympiade St. Moritz 1948, S. 4.

120 Gend. Posten Seefeld an die Staatsanwaltschaft, 8.11.1948, TLA, BH Innsbruck, Entnazifizierung, Karton 724/28, Akt 2554/48.

121 Flaig, Skikanonen, o. S. 
ßig (über-)forderte. ${ }^{122}$ Der Alpinpublizist und Alpenvereinsführer Walther Flaig habe laut Angaben seines Sohnes Günther als ehemaliger Soldat des Ersten Weltkriegs „im Nationalsozialismus eine Hoffnung gesehen“. ${ }^{123}$ Diese Hoffnung kam wiederum in seiner NS-verherrlichenden Bergsteigerlyrik zum Ausdruck. Damit war zumindest eine familiäre Vorprägung gegeben. Immerhin wandte sich Günther Flaig ebenso wie sein Vater der publizistischen Tätigkeit zu und veröffentlichte Ski- und Bergbücher, deren Botschaft nach 1945 noch unverkennbar war. Im Zweiten Weltkrieg verfasste Günther Flaig pathetische Nachrufe wie jenen vom abgestürzten NS-Skistar und Jagflieger Josef Jennewein, ${ }^{124}$ während sein Vater Walther Flaig zunächst noch die „Kampfgemeinschaft“ der aus Österreich stammenden Gebirgsjäger unter dem Kommando von General Eduard Dietl lobte, ehe er von der Österreichischen Alpenzeitung im Juni 1941 neben anderen prominenten Klubkollegen als Leutnant für den Russlandfeldzug gefeiert wurde. ${ }^{125}$ Nach Kriegsende fanden Vater und Sohn wieder zusammen. Die beiden traten nach 1945 sogar einmal gemeinsam im Radio auf. Im Februar 1947 berichteten Günther und Walther Flaig unter dem Titel „Wer wird siegen?“" vorab über die österreichischen Skimeisterschaften in Schruns-Tschagguns. ${ }^{126}$

122 Vgl. Edith Hessenberger, (Keine) Frauen in der Silvretta. Die Ausnahmebergsteigerin Hermine Flaig, in: Michael Kasper/Martin Korenjak/Robert Rollinger/Andreas Rudigier (Hg.), Alltag-Albtraum-Abenteuer. Gebirgsüberschreitung und Gipfelsturm in der Geschichte, Wien/ Köln/Weimar 2015, S. 273-283, hier S. 278-280.

123 Interview mit Günther Flaig, geführt am 2.1.2009. Zit. nach Michael Kasper, Edelweiß und Hakenkreuz? Alpinismus und Nationalsozialismus im ländlichen Raum, in: Edith Hessenberger/Andreas Rudigier/Peter Strasser/Bruno Winkler (Hg.), Mensch \& Berg im Montafon. Eine faszinierende Welt zwischen Lust und Last, Schruns 2009, S. 117-146, hier S. 128.

124 Vgl. Salzburger Zeitung, 27.9.1943, S. 4.

125 Vgl. Amstädter, Der Alpinismus, S. 486 und 496. Eduard Dietl, geboren am 21.7.1890 in Bad Aibling, war Generaloberst der Gebirgstruppen und kommandierte 1940 Teile der 3. Gebirgsdivision bei ihrer Besetzung von Narvik, an der eine Reihe österreichischer Gebirgsjäger beteiligt waren. Dietl bekam von Hitler den Beinamen „Held von Narvik“ verliehen und wurde als erster Offizier mit dem Eichenlaub zum Ritterkreuz des Eisernen Kreuzes ausgezeichnet. Der DAV verlieh Dietl die Ehrenmitgliedschaft und rief einen „Narvik-Wanderpreis“ für Skiwettkämpfe der Gebirgsjäger der Narvik-Division ins Leben. 1941 kommandierte Dietl den Vorstoß von Finnland Richtung Murmansk und ab 1942 war er Oberbefehlshaber der 20. Gebirgsarmee in Norwegen. Er galt als einer der rücksichtslosesten Offiziere des Zweiten Weltkriegs. Dietl starb im Juni 1944 bei einem Flugzeugabsturz. Vgl. Amstädter, Der Alpinismus, S. 485-486; Klee, Personenlexikon, S. 109-110.

126 Vgl. Vorarlberger Volksblatt, 12.2.1947, S. 4. 


\subsubsection{Der Archivar Walther Flaig und das verschwundene Gedächtnis}

Im selben Jahr, als Günther Flaig seine Skifibel über den österreichischen Skisport herausbrachte, war auch sein Vater, scheinbar rehabilitiert von seiner nationalsozialistischen Vergangenheit, vom ÖSV beauftragt worden, das skihistorische Archiv für den Skiverband aufzubauen und zu leiten. ${ }^{127}$ Den Antrag auf der sechsten ÖSV-Länderkonferenz am 6. und 7. Juni 1947 in Heiligenblut stellte Walther Flaig selbst. Laut Protokoll beschloss der ÖSV daraufhin, ein skihistorisches Archiv zu begründen und den Schriftsteller Flaig mit dessen Betreuung zu beauftragen. Beide Beschlüsse erfolgten einstimmig. Das Archiv sollte mit einem jährlichen Etat über 600 Schilling ausgestattet sein. Das Bundesministerium für Unterricht unterstützte das Vorhaben und sagte seine finanzielle Hilfe in Form von Subventionen zu, sollten größere Beträge notwendig sein. ${ }^{128}$ Mitte Juni 1947 begann Flaig mit dem Aufbau des Archivs und stellte seine eigene Sammlung als Leihgabe zur Verfügung. In einem ersten Schritt legte der Leiter des Skihistorischen Archivs (SHA) ein Karteikartensystem an, in dem die Sammlungen verschlagwortet und nach Sachgebieten geordnet waren. Über eine Leihgabe gelangten die beiden Ordner mit Dokumenten zu Hannes Schneider in das Archiv, ${ }^{129}$ die heute noch Teil der Sammlung sind. Der ehemalige Nationalsozialist Flaig prägte fortan das skihistorische Gedächtnis des ÖSV. In einem seiner ersten Schritte verlautbarte er, dass das Skihistorische Archiv des ÖSV alle Druckschriften sammelt, während das Museum in Mürzzuschlag alle anderen Dinge zu sammeln habe. ${ }^{130}$ Dieser Vorschlag dürfte nur zum Teil beherzigt worden sein, da in der Bibliothek von Mürzzuschlag ebenso Druckschriften zu finden sind. Es lässt sich jedoch feststellen, dass es in den nachfolgenden Jahrzehnten zu einem massiven Verlust von Schriftgut im ÖSV-Archiv gekommen ist, der sich aus den Signaturen ablesen und rekonstruieren lässt. So weisen die Bestände des ehemaligen Skihistorischen Archivs des ÖSV in den Jahren zwischen 1938 und 1945 eine durchgehende Lücke auf. Nicht zu finden sind beispielsweise Akten, Protokolle oder Schriftgut des Reichsfachamtes für Skilauf. Ebenso fehlen bis auf wenige Ausnahmen Original-Aufzeichnungen oder Korrespondenzen des ÖSV aus den 1930er-Jahren und für die unmittelbare Nachkriegszeit. Bis heute gibt es keine offizielle Erklärung dafür, wie und wann die

127 Vgl. Skilauf in Österreich. Offizielles Jahrbuch 1948 des Österreichischen Skiverbandes, Wien 1949, S. 40.

128 Vgl. Protokoll, 6. ÖSV-Länderkonferenz in Heiligenblut am 6./7.6.1947, Mappe ÖSV-Länderkonferenz 1946-1948, Archiv Tiroler Skiverband, Kopie im Besitz des Verfassers.

129 Vgl. Skilauf in Österreich. Offizielles Jahrbuch 1948, S. 40.

130 Protokoll, 7. Länderkonferenz in Bad Aussee, 21.9.1947, Archiv Tiroler Skiverband, Kopie im Besitz des Verfassers. 
Bestände verschwunden sind. Die Besetzung von Walther Flaig im Jahr 1947 als Verantwortlichen für das Skihistorische Archiv des ÖSV in diesem Kontext wirft die Frage auf, ob er bewusst belastendes Material skartieren ließ.

Flaig war aber nicht nur der Zuständige in Archivfragen, der in Bludenz wohnhafte Publizist war ebenso für die Pressearbeit des Skiverbandes verantwortlich. Im Juni 1947 beauftragte er die Landespressereferenten der jeweiligen Landesskiverbände mit den Redaktionen zu Beginn der Wintersaison persönlich in Kontakt zu treten. Im Protokoll ist der auf der Länderkonferenz 1947 von Walther Flaig eingebrachte Antrag zur „Fühlungnahme des ÖSV mit dem Syndikat der Sportjournalisten“ vermerkt. ${ }^{131}$ Dieser genehmigte Antrag verdeutlicht, dass der ÖSV in seiner Pressearbeit nichts dem Zufall überließ und hier frühzeitig versuchte, ein auf individuellen Kontakten basierendes Netzwerk zu JournalistInnen aufzubauen. Mit Walther Flaig konnte der Skiverband auf einen erfahrenen Experten in der Presse- und Medienarbeit zurückgreifen. Ab Februar 1947 lancierte der ÖSV-eigene Informationsdienst eine Sendereihe im Radiosender Rot-Weiß-Rot, in der neben dem erfahrenen Sportredakteur Othmar Hassenberger auch der ÖSV-Schriftleiter Walther Flaig zu Wort kam. ${ }^{132}$ Die Sendung trug den klingenden Namen: „Das Skiparadies Österreich“. 133

Sein Sohn Günther Flaig sprach in weiterer Folge aufgrund der Misserfolge der österreichischen SkisportlerInnen in St. Moritz und Mürren von einer „bitteren Lehre“. ${ }^{134}$ Gleichzeitig glaubte er die „alpine Elite“ skitechnisch an einer Wende, die vor allem durch die junge Generation getragen werden sollte. $\mathrm{Zu}$ dieser zählte Flaig den ehemaligen SS-Skiläufer Engelbert Haider. ${ }^{135}$ Laut einer medial veröffentlichten Rangliste der österreichischen Spitzenläufer zu Saisonende 1947 stand Haider in der alpinen Disziplin nach Edi Mall, Hans Nogler, Eberhard Kneissl mit 85 Punkten an vierter Stelle. Die Rangliste des ÖSV-Sportschriftleiters Carl Krois war auf Basis der Rennergebnisse 1946/47 errechnet

131 Vgl. Protokoll, 7. Länderkonferenz in Bad Aussee, 21.9.1947, Archiv Tiroler Skiverband, Kopie im Besitz des Verfassers.

132 Othmar Hassenberger fungierte für das Österreichische Sport-Jahrbuch der austrofaschistischen Sport- und Turnfront als Schriftleiter und publizierte während des Nationalsozialismus weiterhin Sportperiodika, so gab er 1940 unter Mitwirkung der Sportbereichsführung der „Ostmark“ des NSRL das „Sport-Taschenbuch der Ostmark“ heraus. Vgl. u.a. Österreichisches Sport-Jahrbuch. Handbuch für Sport und Turnen. Amtliches Jahrbuch der Österreichischen Sport- und Turnfront, Wien 1937; Othmar Hassenberger (Hg.), Sport-Taschenbuch der Ostmark, Wien 1940.

133 Vgl. Neue Zeit, 6.2.1947, S. 4.

134 Flaig, Skikanonen, o. S.

135 Vgl. Flaig, Skikanonen, o. S. 
worden. ${ }^{136}$ Die Darstellung entsprach sicher nicht der tatsächlichen individuellen sportlichen Leistungsstärke der einzelnen Athleten, wurde aber durchaus als Gradmesser herangezogen.

\subsection{Reintegration im Sportbetrieb}

Der FIS-Kongress in Venedig 1951 bedeutete für den österreichischen Skisport einen weiteren internationalen Durchbruch. Mit Gottfried „Friedl“ Wolfgang (Abfahrt und Slalom), Alfred Rössner (Sprunglauf) und Erika Mahringer (Damen) hatte der ÖSV erstmals in jedem technischen Komitee jemanden positioniert. Außerdem wurde auf der Jahresversammlung der kommende FIS-Kongress für das Jahr 1953 nach Innsbruck-Igls vergeben. ${ }^{137}$ Damit kamen die führenden VertreterInnen des internationalen Skiverbandes erstmals nach 1945 in Österreich zusammen, um dort die künftigen Bestimmungen im Reglement zu besprechen und zu beschließen. Nach Frankreich (1946), Oslo (1949) und Venedig (1951) hatte Österreich in einer knappen Mehrheitsentscheidung den $\mathrm{Zu}$ schlag bekommen. 60 Delegierte aus 19 Mitgliedsländern der FIS kamen beim Kongress in Innsbruck zusammen und beschlossen richtungsweisende Änderungen im Skirennsport, unter anderem die Wiederaufnahme der alpinen Kombination im Wettbewerbsprogramm. ${ }^{138}$

Das gesteigerte Interesse am Skisport und das Engagement des ÖSV auf internationaler Ebene benötigte ExpertInnen und verdeutlicht, warum frühere Spitzenathleten und Skisportfunktionäre, die aufgrund ihrer nationalsozialistischen Vergangenheit zuvor als „belastet“ eingestuft wurden, nun in verschiedenen Bereichen des Sportbetriebes oder in sportnahen Betätigungsfeldern Fuß fassen konnten.

136 Vgl. Rangliste der österreichischen Spitzenläufer, Saisonende 1947, in: Flaig, Skikanonen, o. S. Der Redakteur Carl Krois war nach 1945 Sportschriftleiter des ÖSV und gab unter anderem die Jahrbücher des ÖSV Skilauf in Österreich heraus. Er publizierte Beiträge und sprach im Radio über den österreichischen Skirennsport. Vgl. u. a. Skilauf in Österreich. Offizielles Jahrbuch 1948; Kitzbüheler Anzeiger, 5.2.1977, S. 5; Neue Zeit, 6.2.1947, S. 4.

137 Vgl. Ski Sport, 1 (1952/53) 5/6, S. 4.

138 Vgl. $19^{\text {th }}$ International Ski Congress: 27th to 30th May 1953 - Igls (AUT), https://fisc-webprod.corebine.com/en/inside-fis/about-fis/meetings/fis-congress-history/1953-igls-aut (23.9.2019). 


\subsubsection{Die internationale Nachkriegskarriere von Alfred Rössner}

Einen Einblick, wie vielfältig die Tätigkeitsbereiche für belastete NS-Topathleten nach einer geglückten Rückkehr in den österreichischen und internationalen Skibetrieb sein konnten, gibt der Lebenslauf von Alfred Rössner. Der Spitzenlangläufer Rössner war laut Wehrstammblatt nicht nur ab 1931 Mitglied der SA, sondern trat 1938 auch der NSDAP bei. 1939 wurde er zum SA-Sturmführer befördert und drei Jahre später zum SA-Obersturmführer. ${ }^{139}$ Rössner trainierte 1939 ebenso wie Bradl die HJ. Während des Zweiten Weltkriegs leitete er unter anderem Reichsauswahlkurse auf dem Arthurhaus am Hochkönig und schulte als Heeresbergführer die Soldaten an der Heereshochgebirgsschule in Fulpmes für den Hochgebirgskampf. Bis Ende Mai 1947 hielt sich Rössner in Bad Gastein, danach in Innsbruck und Salzburg auf, wo er offiziell gemeldet war. Der ÖSV bestellte Rössner für die Olympischen Spiele in St. Moritz 1948 zum Trainer der österreichischen Olympiamannschaft, nachdem er 1947 aus dem Lager Glasenbach entlassen worden war. Der Mittelschulprofessor und geprüfte Sportlehrer fuhr danach bis zu seiner Untersuchungshaft im Rahmen des Volksgerichtsverfahrens zwischen den einzelnen Trainingslagern hin und her und konnte vorerst von den Behörden nicht ausfindig gemacht werden. Nach seiner Entlassung aus dem Gefangenenhaus des Landesgerichts Graz zog er 1948 in die Nonntaler Hauptstraße 49 in Salzburg und war bei der Firma Klinserer beschäftigt. Laut amtlicher Häuserkartei war Rössner am 4. August 1948 von Innsbruck zugezogen. ${ }^{140}$ In den Ergebnislisten wurde er aber schon zuvor als Salzburger geführt. Bei den Akademischen Skimeisterschaften Ende Februar 1948 in Schladming wurde Rössner bei den Altakademikern als Salzburger gewertet und konnte nicht einmal ein Jahr nach seiner Entlassung aus dem US-Internierungslager Glasenbach über die 16-Kilometer-Strecke den Sieg bei den Senioren holen. ${ }^{141}$ Bei den österreichischen Skimeisterschaften in Gastein landete Rössner ein paar Tage zuvor auf dem achten Platz. ${ }^{142}$ Das Teilnehmerfeld bei den Senioren war durchaus prominent besetzt und bestand aus ehemaligen SS-Spitzensportlern. Neben Rössner fanden sich noch Paul Haslwanter und Walter Pesentheiner in den Ergebnislisten auf den vorderen Rängen wieder. Pesentheiner war ab März 1938 Gestapo-Beamter und schaffte es innerhalb der SS bis zum Oberscharführer. Er meldete sich im weiteren Kriegsverlauf zur Waffen-SS. Paul Haslwanter diente ab 1938 als SS-Rottenführer im SS-Sturm 3/87 in Inns-

139 OÖLA, LG Linz, Sondergerichte, Sch. 516, VgVr 1049/49.

140 Vgl. Stadtarchiv Salzburg, Häuserkartei, Alfred Rössner.

141 Vgl. Salzburger Volkszeitung, 1.3.1948, S. 4.

142 Vgl. Salzburger Volkszeitung, 19.2.1948, S. 3. 
bruck. ${ }^{143}$ Beide Skiläufer hatten sich nach 1945 einem Entnazifizierungsverfahren zu stellen, ${ }^{144}$ fanden aber, wie Teilnahme- und Ergebnislisten belegen, schnell und ohne größere Hindernisse in den nationalen und internationalen Spitzensport zurück. Ähnlich erging es Rössner, der seine sportliche Heimat mit dem Umzug nach Salzburg 1948 im Skiclub Salzburg fand. Dort engagierte sich Rössner für den Aufbau des Vereins und leistete einen wesentlichen Beitrag in der Jugendarbeit, die von der amerikanischen Besatzungsmacht unterstützt wurde. ${ }^{145}$ Im Salzburger Landesskiverband übernahm Rössner im Herbst 1948 die Agenden des Sportwartes. Als solcher machte er auf den Nachholbedarf des Salzburger Spitzenskisports aufmerksam. ${ }^{146}$ Ab der Wintersaison 1952/53 war Rössner stellvertretender Vorsitzender des Salzburger Landesskiverbandes. Als Sportwart für die nordischen Bewerbe, Rössners Spezialgebiet, wurde ein anderer ehemaliger NS-Sportfunktionär eingesetzt, den Rössner von früher kannte, der in Bischofshofen lebende Anton Höttl. ${ }^{147}$

Rössner bekräftigte in seinem Gnadengesuch an den Bundespräsidenten im Dezember 1950 seine Bemühungen um den österreichischen Skisport. In dem Schreiben führte er nicht nur seine guten Kontakte zum internationalen Skiverband (FIS) ins Feld, sondern auch seine sportlichen Erfolge bei den nachträglich annullierten Kriegs-Ski-Weltmeisterschaften in Cortina D’Ampezzo 1942. Als Trainer stehe ihm außerdem ein „großer Teil der Erfolge“ im österreichischen Nachkriegsskisport zu, diese seien durchschlagend gewesen, „insbesondere auch für die Werbung und Förderung des österr. Fremdenverkehrs“. ${ }^{148} \mathrm{Er}$ sei zudem der einzige, „der praktisch und theoretisch den gesamten sportlichen Skilauf in Österreich leiten kann“. ${ }^{149}$ Die Beförderung zum SA-Obersturmführer gehe auf seine sportlichen Leistungen zurück. Wie andere sei er als Aushängeschild der SA-Formation benützt worden. 1951 wurde das Volksgerichtsverfahren wegen §11-Verbotsgesetz gegen Rössner eingestellt.

143 Vgl. dazu TLA, BH-Akten und BPD Innsbruck NS-Dokumentationsmaterial 3/237, Erfassung der SS-Angehörigen.

144 Paul Haslwanter und Walter Pesentheiner mussten sich einem Volksgerichtsverfahren unterziehen. Vgl. TLA, BH Innsbruck, Entnazifizierung, Karton 724/28, Akt 2554/48.

145 Vgl. u. a. Ski-Klub Salzburg, 100 Jahre, S. 27.

146 Vgl. Salzburger Nachrichten, 29.11.1948, S. 3.

147 Vgl. Ski Sport 1 (1952) 1, S. 10.

148 Abolitionsgesuch Alfred Rössner, Salzburg, 13.12.1950, OÖLA, LG Linz, Sondergerichte, Sch. 516, VgVr 1049/49.

149 Abolitionsgesuch Alfred Rössner, Salzburg, 13.12.1950, OÖLA, LG Linz, Sondergerichte, Sch. 516, VgVr 1049/49. 


\subsubsection{Rössners Bestellung zum ÖSV-Cheftrainer und ÖSV-Sportwart}

Dass Rössner vollständig rehabilitiert wurde, zeigt seine Anstellung als ÖSVCheftrainer für das Olympiajahr 1952. Neben der Langlauf-Staffel übernahm Rössner das Training der alpinen Herren-Nationalmannschaft. Als solcher trainierte er unter anderem den späteren Olympia-Sieger Toni Sailer im so genannten „weißen Wunderteam“. Zunächst war Rössner aber bei den Olympischen Spielen 1952 in Oslo erfolgreich, als die österreichischen Skisportler fünf von neun möglichen Olympia-Medaillen gewinnen konnten. Rössner hatte sich im Laufe seiner Karriere ein umfassendes Wissen in der Trainingslehre angeeignet und war nun als erster Sportwart des ÖSV offiziell beauftragt, dieses im Rennsport umzusetzen. In der ab Mitte November 1952 erscheinenden ÖSV-Zeitschrift Ski Sport veröffentlichte Rössner sporttheoretische Beiträge zur Aufbauarbeit im alpinen und nordischen Skisport. ${ }^{150}$ Als Vorbilder dienten die beiden ehemaligen NSRL- und nunmehrigen ÖSV-Spitzenathleten Josef Bradl und Hellmut Lantschner.

Nach den Erfolgen bei den Olympischen Winterspielen 1956 - drei Goldmedaillen für Toni Sailer - kam es zu Meinungsverschiedenheiten mit ÖSV-Athleten aus dem Arlberger Ski-Umfeld. ${ }^{151}$ Diese kritisierten Rössners Vorgehen. Rössner kümmerte sich aber nicht weiter darum, er verfolgte bereits andere Pläne. Der darauffolgende Vorsitz im Salzburger Landesskiverband von 1956 bis $1958^{152}$ war nur eine Zwischenstation.

\subsubsection{Rössners Rückkehr auf das internationale Sportparkett}

Zwei Jahre nachdem das Volksgerichtsverfahren gegen Rössner eingestellt worden war, nahm er Ende Mai 1953 am internationalen FIS-Kongress in Innsbruck teil. Ein Foto zeigt ihn gemeinsam mit dem ÖSV-Funktionär Hans Hartwagner, dem 1936 in die USA ausgewanderten Skisportler Sepp Ruschp, ${ }^{153}$ und der Inns-

150 Vgl. Ski Sport 1 (1952) 1, S. 5-7; Ski Sport 1 (1952) 2, S. 6-7.

151 Vgl. Alfred Rössner 94-jährig gestorben, https://www.derstandard.at/story/2288175/alfred-roessner-94-jaehrig-gestorben (30.8.2019).

152 Vgl. Landessportorganisation Salzburg (Hg.), 25 Jahre Landessportorganisation. Ein Vierteljahrhundert in Bewegung, Salzburg 1972, S. 58.

153 Sepp Ruschp migrierte auf Einladung des Mount Mansfield Ski Club in Stowe im Dezember 1936 nach Vermont. Der gebürtige Linzer arbeitete als Skilehrer und unterrichtete an der University of Vermont und Norwich. Ruschp wurde 1978 in die United States Ski Hall of Fame aufgenommen. Er starb 1990 im Alter von 81 Jahren. https://skihall.com/hall-of-famers/seppruschp/ (30.8.2019). 
brucker Skirennläuferin Erika Mahringer. ${ }^{154}$ Für den FIS-Kongress in InnsbruckIgls war auch der 1939 vom NS-Terror geflüchtete Hannes Schneider nach Europa gereist. Ein Foto zeigt Schneider bei dem FIS-Kongress neben Ruschp sitzend. ${ }^{155}$

Rössner war für sein Wissen rund um die Trainingslehre sowohl national als auch international gefragt und anerkannt. In Sportkreisen galt er als „Universal-Genie“ und „Alleskönner“. ${ }^{156}$ In Zeiten des Kalten Krieges schaffte er es sowohl im Westen als auch im Osten seine Kontakte und ein gut funktionierendes Netzwerk aufzubauen. Nicht einmal ein Jahr nachdem der österreichische Staatsvertrag im Mai 1955 unterzeichnet worden war, fragte die ÖsterreichischSowjetische Gesellschaft am 21. März 1956 im Namen des sowjetischen Kulturattachés bei Rössner an, ob eine Möglichkeit der sportlichen Zusammenarbeit bestehe. Konkret sollte Rössner den sowjetischen Skisportlern das österreichische System näherbringen. Angedacht waren gemeinsame Trainingseinheiten in Theorie und Praxis bis hin zum Austausch von Trainern. ${ }^{157}$ Rössner antwortete prompt und schrieb drei Tage später zurück, dass er schon lange der Überzeugung sei, dass die Zusammenarbeit auf dem Gebiet des alpinen Skisportes intensiviert werden müsste. Leider stehe er mit dieser Ansicht im Verband ziemlich allein da. Rössner schrieb weiter:

So wie ich aber bisher viele andere Nationalmannschaften beraten habe - die Skandinavier, Amerikaner, Polen, Jugoslaven, einzelne schweizerische Läufer usw. würde ich selbstverständlich auch die russischen alpinen Läufer bezw. ihre Trainer beraten. ${ }^{158}$

Einem gemeinsamen Training erteilte Rössner im Vorhinein die Absage, da dieses weder organisatorisch noch technisch umsetzbar sei. Er erklärte sich aber für ein Gespräch bereit, dieses sollte jedoch vor einem rein fachlichen Hintergrund stattfinden und keinen politischen Hintergrund haben. Rössner hatte

154 Vgl. Privatnachlass Hans Hartwagner, Foto FIS-Kongress Innsbruck 1953 mit Hans Hartwagner, Ahrer, Sepp Ruschp, Erika Mahringer, Alfred Rössner und Steiner, Kopie im Besitz des Verfassers.

155 Schneider war 1953 nach Europa gereist und hielt sich nach dem FIS-Kongress noch in St. Anton auf. Vgl. Privatnachlass Hans Hartwagner, Foto FIS-Kongress Innsbruck 1953 mit Hannes Schneider und Sepp Ruschp; Thöni, Hannes Schneider, S. 139.

156 Vgl. Alfred Rössner 94-jährig gestorben, https://www.derstandard.at/story/2288175/alfred-roessner-94-jaehrig-gestorben (30.8.2019).

157 Vgl. Privatnachlass Alfred Rössner, Brief von M. Grünberg, Sekretär der ÖsterreichischSowjetischen Gesellschaft in Wien an Fred Rössner, Wien, 21.3.1956, Sammlung Privatnachlässe, Landesskimuseum Werfenweng.

158 Privatnachlass Alfred Rössner, Brief von Fred Rössner an die Österreichisch-Sowjetische Gesellschaft, Salzburg, 24.3.1956, Sammlung Privatnachlässe, Landesskimuseum Werfenweng. 
demnach keine ideologischen Bedenken, so lange die Zusammenarbeit auf einer sportlichen Ebene blieb.

Als FIS-Delegierter nahm Rössner in weiterer Folge an den internationalen FIS-Kongressen teil. So auch 1977 beim Internationalen FIS-Kongress in San Carlos de Bariloche in Argentinien, dort traf er auf altbekannte Gesichter aus seiner aktiven Zeit als Sportler.

Im September 1985 wurde Rössner vom österreichischen Hochschulausschuss aufgrund seiner Leistungen für den Studentensport zum Ehrenmitglied des internationalen Hochschulsportverbandes (FISU) ernannt. In einem Schreiben im Zuge der nordischen Skiweltmeisterschaften in der Ramsau bedankte sich ÖSV-Präsident Peter Schröcksnadel im Mai 1999 bei Rössner für seine Verdienste für den österreichischen Langlaufsport und bezeichnete ihn als „Vater des Langlauf-Sports in Österreich“ “. ${ }^{159}$ Rössner starb am 25. Dezember 2005 in Salzburg. ${ }^{160}$

\subsubsection{Die Rückkehr der SS-Sportler Gregor Höll und Engelbert Haider in den Trainings- und Sportbetrieb}

Gemeinsam mit Rössner kehrten auch andere ehemalige NS-Spitzenathleten, die ab 1938 und teilweise schon zuvor in der SA oder SS Karriere gemacht hatten, auf die sportliche Bühne zurück. Ihre skisportlichen Kenntnisse wurden mitunter für die Ausbildung des Skinachwuchses herangezogen und geschätzt. So trafen Fred Rössner, Engelbert Haider und Gregor Höll im Dezember 1948 am Hochkönig zusammen, um dort gemeinsam mit der Salzburger Nachwuchshoffnung Annelore Zückert die Trainingskurse für Wettläufer zu leiten. ${ }^{161}$ Gregor Höll, der während des Zweiten Weltkriegs für die SS-Sportgemeinschaft an den Start ging und im Wehrmachtsausbildungsstab der $\mathrm{HJ}$ stand, war zumindest bis Ende Juli 1947 im Camp Markus W. Orr interniert. Das geht aus einer Teilnehmerliste eines Massagekurses hervor, an dem Höll neben anderen inhaftierten Skiathleten im Sommer 1947 teilnahm. Der Massagekurs fand im Rahmen der von der amerikanischen Militärregierung genehmigten und vom „Educational Service“ angebotenen Umschulungskurse im Internierungslager statt. Geplant war eine viermonatige Ausbildung, die aus zwei Monaten Theorie und zwei Monaten Praxis bestand. Den berufsbildenden Umschulungskurs zum Heilmasseur

159 Vgl. Privatnachlass Alfred Rössner, Brief von ÖSV-Präsident Peter Schröcksnadel an Fred Rössner, Innsbruck 11.5.1999, Sammlung Privatnachlässe, Landesskimuseum Werfenweng.

160 Vgl. u. a. Ski-Klub Salzburg, 100 Jahre, S. 60.

161 Vgl. Oberösterreichische Nachrichten, 25.11.1948, S. 3. 
leitete der Lagerarzt Dr. Erwin Risak. ${ }^{162}$ Der bekannte Wiener Internist war aufgrund seiner SS-Zugehörigkeit, Risak war mit Stand 1942 SS-Obersturmführer, selbst interniert. ${ }^{163}$ Risak war aber kein gewöhnlicher Häftling, er leitete als anerkannte Koryphäe das Lagerspital. ${ }^{164}$ Höll bekam also die Gelegenheit, sich von dem belasteten Herzspezialisten Risak zum Heilmasseur ausbilden zu lassen. Im Lager Glasenbach traf Höll auf seinen Skisprungkollegen Josef Bradl und andere ehemalige SA- und SS-Skisportler. ${ }^{165}$ So befand sich unter den angemeldeten Teilnehmern für den Massagekurs der in Salzburg geborene ehemalige SS-Offizier und NSRL-Kreisbeauftragte für den Pongau Siegfried Amanshauser. ${ }^{166}$ Ab September 1946 fanden im US-amerikanischen Internierungslager wöchentlich Sportveranstaltungen in verschiedenen Sportarten statt, deren Zahl in die Hunderte ging. ${ }^{167}$ Für Höll und seine Sportkameraden bestand also durchaus die Möglichkeit, sich fit zu halten und sich auf die Zeit nach der Inhaftierung sportlich wie beruflich vorzubereiten. Im Winter 1947/48 kehrte Höll nach seiner Internierung erfolgreich in den Spitzensport zurück, ohne dass sich der SS-Oberscharführer einem Entnazifizierungsprozess stellen musste. ${ }^{168} \mathrm{Im}$ Gegensatz zu Bradl wurde Höll zu den Olympischen Spielen in St. Moritz 1948 zugelassen und wurde beim Mannschaftsempfang am 16. Jänner 1948 von Bundespräsident Karl Renner im Wiener Rathaus angelobt. ${ }^{169}$ Höll landete beim Skispringen als zweitbester Österreicher hinter Hubert Hammerschmidt auf Platz 24. Nach den Olympischen Spielen duellierte sich Höll bei den Österreichischen Meisterschaften in Bad Hofgastein im März 1948 mit Josef Bradl im Springen

162 Vgl. SLA, Camp Marcus W. Orr 1946/47, diverse Unterlagen.

163 Vgl. Klee, Personenlexikon, S. 499; Margit Reiter, Die Ehemaligen. Der Nationalsozialismus und die Anfänge der FPÖ, Göttingen 2019, S. 43.

164 Vgl. Oskar Dohle, Sport im „Lager Glasenbach“, in: Minas Dimitriou/Oskar Dohle/Walter Pfaller/Andreas Praher (Hg.), Salzburgs Sport in der NS-Zeit. Zwischen Staat und Diktatur, Salzburg 2018, S. 329-334, hier S. 330.

165 Josef Bradl war ebenfalls bis 1947 im Lager Glasenbach inhaftiert. Vgl. SLA, NS-SOKO A02.04 Bo-Br.

166 Vgl. BArch (ehem. BDC), SSO, Amanshauser, Siegfried, 15.5.1895; SLA, Camp Marcus W. Orr 1946/47, diverse Unterlagen.

167 Der Historiker Oskar Dohle hat für das Forschungs- und Dokumentationsprojekt Salzburgs Sport in der NS-Zeit die zahlreichen Sportveranstaltungen und Trainingsmöglicheiten im Camp Markus W. Orr für die Jahre 1946 bis 1947 statistisch erfasst. Vgl. Dohle, Sport im Lager, S. 332333.

168 Ein Gregor Höll, geboren am 16.6.1911 in Lungötz, taucht weder in den Akten der NS-Sonderkommission des SLA noch in den Volksgerichtsakten des OÖLA auf. Auch in der zentralen Meldekartei der WASt in Berlin findet sich kein Eintrag zu einem Gregor Höll.

169 Vgl. Privatnachlass Gregor Höll, Programm für den Empfang der Österreichischen Olympia- Wintersportmannschaft, Sammlung Privatnachlässe, Landesskimuseum Werfenweng. 
und wurde Vizemeister. ${ }^{170}$ Beide starteten damals für den ASKÖ Bischofshofen. Bei internationalen Konkurrenzen in Ponte di Legno und Arosa belegte der mittlerweile knapp 37-jährige Höll je einen dritten Platz. 1950 startete er nochmals erfolgreich bei den ÖSV-Meisterschaften und wurde beim Bergisel-Springen in Innsbruck Dritter. Nach seiner aktiven Karriere, die er 1951 beendete, führte er ein Sportartikelgeschäft. ${ }^{171}$

Sein um elf Jahre jüngerer Teamkollege Engelbert Haider konnte wie Höll auf eine nahezu ununterbrochene und lückenlose skisportliche Karriere im Zweiten Weltkrieg zurückblicken und befand sich 1948 auf dem Höhepunkt seiner sportlichen Karriere. Haider wurde immer wieder für wichtige Skisportveranstaltungen freigestellt. Noch im März 1944 nahm er an den reichsdeutschen Skimeisterschaften in den alpinen Bewerben am Arlberg teil und gewann dort den Slalom und die Kombination. ${ }^{172}$ Die französischen Besatzungsbehörden vernahmen Haider 1948 wegen seiner SS-Vergangenheit und seines Einsatzes für den SD in Norwegen. Sie verdächtigten den SS-Sportler, ein Mitglied der Waffen-SS gewesen zu sein. ${ }^{173}$ Das Bundesministerium für Unterricht in Wien gab bereits am 18. November 1946 Entwarnung. In einem Schreiben an den ÖSV und den Tiroler Skiverband in Innsbruck hieß es, dass „weder in politischer noch moralischer Hinsicht etwas Nachteiliges vorliegt. Es besteht daher seitens des Bundesministeriums für Unterricht kein Bedenken gegen den Start Haiders bei Schi-Wettkämpfen““ ${ }^{174}$ Haider war zu diesem Zeitpunkt in Salzburg gemeldet. Im Februar 1951 wurde Haider in den Salzburger Nachrichten als „Abfahrtskönig“ gefeiert. „Aus dem Torlauftalent ist Österreichs bester Abfahrer geworden “, ${ }^{175}$ hieß es in dem Bericht anlässlich der österreichischen Skimeisterschaften im oberösterreichischen Windischgarsten.

\subsubsection{Die Berufung von Anton Seelos zum Nationalteamtrainer}

Die Wiedereinsetzung des „belasteten“ Anton Seelos als Nationaltrainer spielte sich auf ministerieller Ebene ab. Nachdem dieser zuletzt bei einer Hochgebirgskompanie der Ordnungspolizei am Achensee stationiert und von dieser geflüch-

170 Vgl. Salzburger Volkszeitung, 8.3.1948, S. 4.

171 Vgl. Glaser, Goldschmiede, S. 150.

172 Vgl. Völkischer Beobachter, 6.3.1944. S. 6.

173 Vgl. TLA, Wehrstammbuch, Engelbert Haider.

174 Bundesministerium für Unterricht, Wien am 18. November 1946 an den Österreichischen Skiverband und den Tiroler Skiverband in Innsbruck, TLA, Wehrstammbuch, Engelbert Haider. 175 Engele Haider - Abfahrtskönig in: Salzburger Nachrichten, 5.2.1951, Sportbeilage am Montag, o. S. 
tet war, weil er aufgrund seiner angeblichen Widerstandstätigkeit einer Verhaftung entgehen wollte, wurde er zunächst in Haft genommen. Doch schon bald setzte sich das Bundesministerium für Unterricht aus nationalem Interesse für den SA-Sportler und ehemaligen Trainer der Ordnungspolizei-Reichsmannschaft ein. Das zuständige Ministerium unter Unterrichtsminister Felix Hurdes (ÖVP) schrieb am 20. September 1947 an die Tiroler Landesregierung folgende Zeilen:

Toni Seelos ist als Skiläufer von Weltklasse, als Schilehrer und Trainer der Olympiamannschaft für den Österreichischen Skilauf und Fremdenverkehr von größter Bedeutung. Seine österreichische Gesinnung darf als außer Zweifel stehend bezeichnet werden. ${ }^{176}$

Aufgrund dessen sei seine Begnadigung im Sinne des öffentlichen Interesses. Die zuständige Behörde solle daher Nachsicht gewähren, ansonsten könne Seelos die österreichische Olympiamannschaft nicht trainieren, hieß es im November 1947, ein paar Monate vor Beginn der Spiele in St. Moritz. ${ }^{177}$ Seelos selbst wollte mit einem Gnadengesuch dem Anliegen des Bundesministeriums noch Nachdruck verleihen und schrieb von sich in dritter Person:

\begin{abstract}
Er war seit jeher begeisterter Skisportler und hatte für Politik nie etwas übrig. Er war vor und nach dem Jahre 1938 nur Skisportfachmann und hat sich um die politischen Ereignisse überhaupt nicht gekümmert. Nach dem Umbruche 1945 stellte er sich sogleich der Gemeinde Seefeld für den Wiederaufbau des Fremdenverkehrs- und Skisportwesens, sowohl geistig als auch manuell voll und ganz zur Verfügung. Durch diese Tat bewies er, dass er sich für ein unabhängiges Österreich einsetzt und bereits durch seinen freiwilligen Einsatz für den Wiederaufbau im Fremdenverkehrs- und Skisportwesen nicht nur für die Gemeinde Seefeld, sondern auch für ganz Tirol, einen Teil geleistet hat. Sein seinerzeitiger Beitritt zur NSDAP und SA dürfte nur aus Existenzgründen (Skilehrerkonzession) auf Druck der Partei und SA erfolgt sein, da diese ein grosses Aushängeschild benötigten. ${ }^{178}$
\end{abstract}

Die Intervention des Unterrichtsministerium hatte zur Folge, dass Seelos im Winter 1947/48 als Cheftrainer die Vorbereitung der alpinen Olympiamannschaft des ÖSV für St. Moritz übernehmen konnte. Am 2. August 1948 wurde sein Ansuchen um Begnadigung aufgrund der Minderbelastetenamnestie gegenstandslos und Seelos war offiziell dazu berechtigt, das Traineramt des öster-

176 Bundesministerium für Unterricht an Amt der Tiroler Landesregierung, 20.9.1947, TLA, BH Innsbruck, Entnazifizierung, 2592/48.

177 Bundesministerium für Unterricht an Amt der Tiroler Landesregierung, 18.11.47, TLA, BH Innsbruck, Entnazifizierung, 2592/48.

178 Gnadengesuch Anton Seelos, Landesgendarmeriekommando für Tirol an die Sicherherheitsdirektion für Tirol, 26.02.1948, TLA Sonderbehörden nach 1868 - Sicherheitsdirektion für Tirol (ATLR Abteilung VII) - Staatspolizeiliche Akten 1945 - Pos. 2479. 
reichischen Skinationalteams zu übernehmen. ${ }^{179}$ Bei der Hauptversammlung des ÖSV im September 1948 in Radstadt wurde Seelos zum ersten Sportwart gewählt. ${ }^{180}$ Er stand dem ÖSV bis 1956 als Trainer zur Verfügung. Ab dem Winter 1948 machte sich Seelos nicht nur als ÖSV-Nationaltrainer einen Namen, sondern auch als Skiwachs-Hersteller. ${ }^{181}$

\subsubsection{Josef Bradl und der vermeintliche „Sieg für Österreich“}

Der Salzburger Skisprung-Weltmeister Josef Bradl war nach Kriegsende aufgrund seiner (illegalen) SA-Mitgliedschaft bis 17. Jänner 1947 im US-amerikanischen Internierungslager Glasenbach in Salzburg (Camp Marcus W. Orr) inhaftiert und durfte 1948 trotz aller Interventionen bei den Olympischen Winterspielen in St. Moritz nicht starten, weil ihn die Schweizer Behörden nach vorangegangen Protesten Norwegens und Hollands nicht einreisen ließen. Schon 1947 beschäftigte die Olympia-Teilnahme Bradls die österreichischen Behörden. Der Salzburger Landeshauptmann Albert Hochleitner setzte sich bei Innenminister Oskar Helmer dafür ein, dass Bradl trotz seines Dienstranges eines SASturmmanns für seine Reise nach St. Moritz gültige Papiere ausgestellt bekommt. Helmer verwies darauf, dass ein positives Ansuchen des Olympischen Komitees helfen könnte. ${ }^{182}$ Daraufhin versuchte Edgar Fried, Leiter des österreichischen Olympischen Komitees, eine Befürwortung der Teilnahme Bradls beim Olympischen Komitee in St. Moritz zu erwirken. Dieser Versuch blieb erfolglos. Bradl wurde die Einreise in die Schweiz per Telegramm verweigert und der Weltmeister von 1939 war in St. Moritz der große Abwesende während sein Langzeit-Kontrahent und Freund aus Norwegen Birger Ruud als Sieger gefeiert wurde. ${ }^{183}$ Die Salzburger Nachrichten wünschten sich Bradl nach den mäßigen Leistungen der anderen österreichischen Springer und dem norwegischen Dreifachsieg nach St. Moritz und titelten „Bradl hätte Fünfter werden können““ ${ }^{184}$ Stattdessen sprang der Salzburger bei den ASKÖ-Bundesmeisterschaften in Bad Aussee um den Verbandstitel.

Bradl nutzte die verordnete Zwangspause und konzentrierte sich in dieser Zeit aufs Schreiben. Er verfasste seine Autobiografie Mein Weg zum Weltmeister,

179 TLA, BH Innsbruck, Entnazifizierung, 2592/48.

180 Vgl. Skilauf in Österreich. Offizielles Jahrbuch 1948, S. 194.

181 Vgl. u. a. Salzburger Volkszeitung, 3.12.1948, S. 3.

182 Vgl. SLA, PRÄ 1947/09.

183 Vgl. SLA, PRÄ 1947/09; Salzburger Nachrichten, 29.1.1948, S. 2.

184 Salzburger Nachrichten, 9.2.1948, S. 3. 
die in einer ersten Ausgabe 1948 erschien und 1952 neu aufgelegt wurde. ${ }^{185}$ Die zweite Auflage erschien mit einem Vorwort des wiedereingesetzten Salzburger Landesverkehrsamtsdirektors Hans Hofmann-Montanus exakt in jenem Jahr, in dem Bradl bei den Olympischen Spielen 1952 in Oslo wieder an den Start ging. ${ }^{186}$ Bradls schriftstellerischer Beitrag reihte sich in eine Reihe von SportlerInnenbiografien ein, die ab den 1950er-Jahren eine Renaissance erlebten und erstmals zur Vermarktung der AthletInnen eingesetzt wurden. ${ }^{187}$ Interessanterweise trug Toni Sailers Autobiografie Mein Weg zum dreifachen Olympiasieg, geschrieben von Karl Springenschmid 1956 einen zum Verwechseln ähnlichen Titel. ${ }^{188}$ Doch zurück zu Bradl und seiner Autobiografie. In seiner Selbstdarstellung blendet Bradl seine Beteiligung als SA-Sportler am NS-Regime aus. Generell vermeidet er darin, die nationalsozialistische Herrschaft $\mathrm{zu}$ benennen, als hätte es diese von 1938 bis 1945 in Österreich nicht gegeben. ${ }^{189}$ Den Weltmeistertitel 1939 für das Deutsche Reich titulierte Bradl in seiner Autobiografie mit „Sieg für Österreich“. 190

$\mathrm{Zu}$ Beginn der 1950er-Jahre sollte der Mühlbacher, der mittlerweile für den ASKÖ Bischofshofen an den Start ging, zu einer neuen sportlichen Hochform auflaufen. Das Wettkampfjahr 1951 entwickelte sich zu einem der erfolgreichsten in seiner sportlichen Laufbahn. Bei der Skiflugwoche in Oberstdorf im März 1951 stand Bradl mit 124 Metern den weitesten Flug und wurde vor 6000 Zuschauern Tagesbester. Der Empfang, der ihm anschließend vom ASKÖ-Landesverband auf dem Salzburger Hauptbahnhof bereitet wurde, stand jenem zur Zeit des Nationalsozialismus von 1939 um nichts nach. Bradl sei „mit einem Schlage wieder Österreichs populärster und größter Skisportler“, hieß es in den Salzburger Nachrichten. ${ }^{191}$ Einen Tag nach seiner Ankunft überreichte ihm der Salzburger Landeshauptmann Josef Klaus das goldene Sport-Ehrenzeichen des Landes. ${ }^{192}$

185 Vgl. Sepp Bradl, Mein Weg zum Weltmeister, Innsbruck 1948; Sepp Bradl, Mein Weg, 1952. 186 Vgl. hier Geleitwort von Hans Hofmann-Montanus, in: Sepp Bradl, Mein Weg zum Weltmeister, Innsbruck 1952, S. 9-11.

187 Vgl. Volker Kluge, Lebensläufe von Sportlern und Sportfunktionären zwischen Sport, Politik, Kultur, Medien und Gesellschaft, in: BIOS 18 (2005) 2, S. 206-214, hier S. 209.

188 Vgl. Toni Sailer, Mein Weg zum dreifachen Olympiasieg, Salzburg/Stuttgart 1956. Karl Springenschmid, seit 1932 NSDAP-Mitglied, war Leiter des NS-Lehrerbundes im Gau Salzburg und Initiator der nationalsozialistischen Bücherverbrennung 1938 in der Stadt Salzburg. Nach 1945 entzog er sich seiner Verhaftung durch Flucht und wirkte nach Einstellungen der gerichtlichen Ermittlungen 1951 erneut als Schriftsteller. Vgl. u. a. Hofinger, Nationalsozialismus, S. 147 und 161.

189 Vgl. Bradl, Mein Weg, 1948 und 1952.

190 Vgl. Bradl, Mein Weg, 1952, S. 152.

191 Vgl. Salzburger Nachrichten, 1.3.1951, S. 4.

192 Vgl. Salzburger Nachrichten, 7.3.1951, S. 4. 


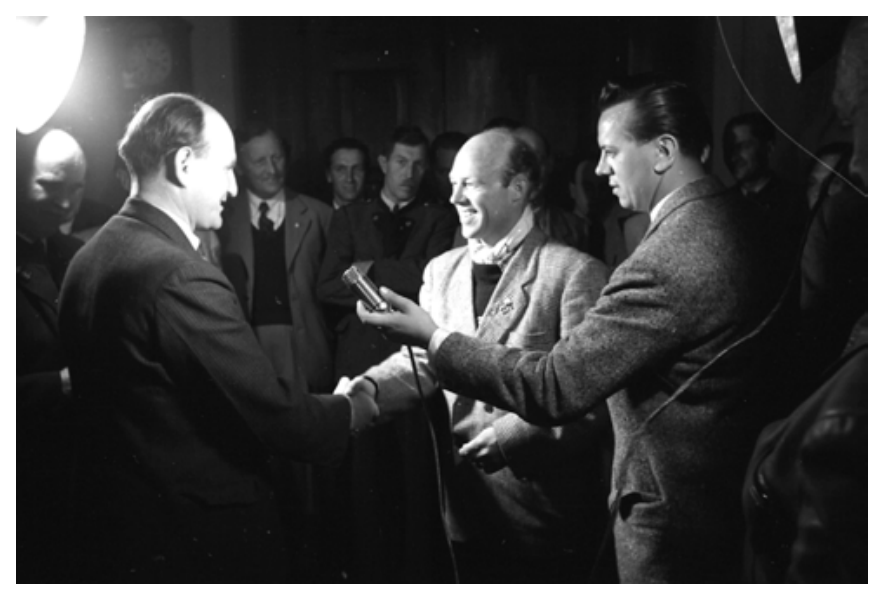

Abb. 39: Der Salzburger Landeshauptmann Josef Klaus übergibt Josef Bradl das Goldene Sport-Ehrenzeichen des Landes Salzburg, 6. März 1951, SLA, LBS F35/0330.

Die Olympia-Teilnahme 1952 in Oslo beendete Bradl mit einem Sturz, dieser begrub seine Hoffnungen auf eine Olympia-Medaille. ${ }^{193}$ Bei der Vier-SchanzenTournee, die im Jänner 1953 in Garmisch-Partenkirchen, Oberstdorf, Innsbruck und Bischofshofen ausgetragen wurde, landete Bradl mit einer Gesamtnote von 878,6 Punkten vor seinen norwegischen Kontrahenten Halvor Næs und Asgeir Doelpads auf dem ersten Platz. ${ }^{194}$ Bei der kommenden Tournee musste er sich angesichts des jungen Skisprungnachwuchses aus Norwegen und Finnland mit einem dritten Platz zufrieden geben. ${ }^{195}$ Bei den darauffolgenden österreichischen Meisterschaften konnte Bradl noch einmal im Spezialsprunglauf aufzeigen und holte dort erneut den Staatsmeistertitel. ${ }^{196}$ Seine aktive Karriere als Skispringer beendete er 1956 nach dem zwölften Platz bei den Olympischen Spielen in Cortina d'Ampezzo. Danach war Bradl noch als Trainer der nordischen Skinationalmannschaft erfolgreich und trainierte die junge österreichische Skisprung-Generation bis in die späten 1960er-Jahre hinein. Der Skisprungstar diente wie schon zur Zeit des Nationalsozialismus als Vorbild für die Sportjugend, dieses Mal für jene der Zweiten Republik. Bradl starb am 3. März

193 Vgl. Glaser, Goldschmiede, S. 55.

194 Vgl. Ski Sport, 1 (1952/53) 3, S. 6.

195 Vgl. Ski Sport, 2 (1953/54) 3, S. 4-5.

196 Vgl. Ski Sport, 2 (1953/54) 4/5, S. 7. 
1982 mit 64 Jahren. 1991 wurde das Skisprungstadion in Bischofshofen nach ihm benannt, das erstaunlicherweise heute noch seinen Namen trägt. ${ }^{197}$

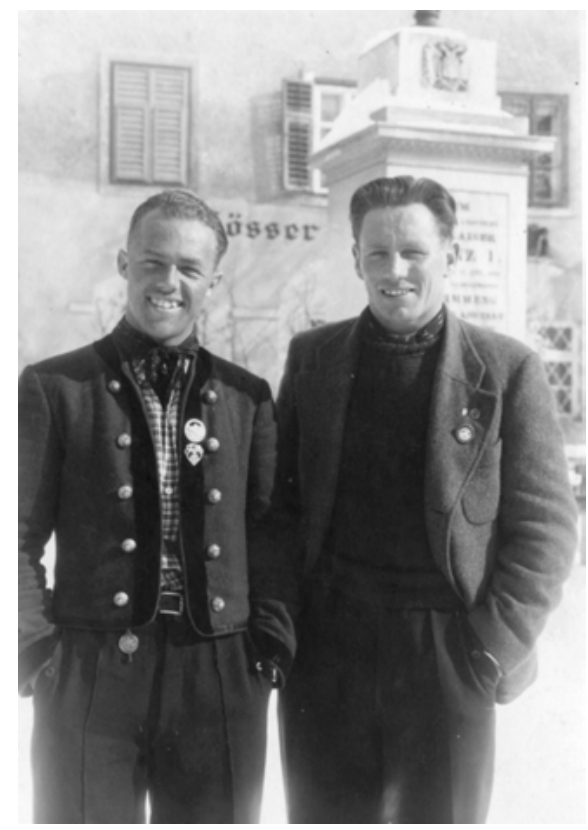

Abb. 40: Wiedersehen nach dem Krieg: Der frühere SA-Sturmführer Josef Bradl (li.) und der rehabilitierte SS-Sportsoldat Wilhelm Köstinger bei einem Treffen in Gastein, Privatbesitz.

Ebenso wie Bradl hatte sich sein langjähriger Weggefährte Markus Maier nach 1945 vor der NS-Sonderkommission zu verantworten. Die Argumente waren ähnliche, wie sie auch andere belastete und minderbelastete Skisportler im Entnazifizierungsverfahren vorbrachten:

Lediglich aus dem Grunde der sportlichen Repräsentation wurde ich als bekannter Skirennfahrer- und Springer im Feber 1940, anläßlich der SA-Gruppenskiwettkämpfe in Gastein zum SA-Sturmführer gemacht, weil nach den bestehenden Vorschriften nur ein Offiziersdienstgrad der SA als Skimannschaftsführer verwendet werden durfte. ${ }^{198}$

Mit Hilfe dieser Argumentation konnte Maier seine SA-Mitgliedschaft und damit seine Beteiligung am NS-System entkräften. Bei Julius Funcke, der bereits vor 1938 maßgeblich am Aufbau der illegalen SA im Salzburger Lungau beteiligt war, verhielt es sich ähnlich. Der Skilehrer Funcke gab zwar im Volksgerichts-

197 Vgl. Glaser, Goldschmiede, S. 55; Praher, Vergessen und verdrängt, S. 369-370; Egon Theiner, Sepp Bradl. Der Adler vom Hochkönig, in: Matthias Marschik/Georg Spitaler (Hg.), Helden und Idole. Sportstars in Österreich, Innsbruck/Wien/Bozen 2006, hier S. 205-206.

198 Vgl. SLA, NS-SOKO A.04.11 B, Markus Maier. 
verfahren zu, 1932 der SA beigetreten und als illegaler Nationalsozialist tätig gewesen zu sein, betonte aber gleichzeitig: „Es ist entschieden zu viel gesagt dass ich Fanatiker gewesen wäre, ich war wohl damals jung, war für allen Unfug zu haben, habe aber politisch mich wenig interessiert.“199 Offiziell als „minderbelastet“ eingestuft, konnte der ehemalige SA-Truppführer seine Bergleidenschaft nach dem Zweiten Weltkrieg bei der Alpinen Bergrettungsstelle am Prebersee ausleben, ${ }^{200}$ dort, wo seine Karriere in den 1930er-Jahren als Skilehrer begann und seine Annäherung an den Nationalsozialismus seinen Ausgang nahm.

Auf Funktionärsebene gelangte der im November 1944 verstorbene NS-Landessportführer für Vorarlberg, NSDAP-Kreisorganisationsleiter und NS-Gesinnungsgenosse Theodor Rhomberg posthum zu Ehren. Er erhielt in der Nachkriegszeit das „Goldene Ehrenzeichen“ des ÖSV verliehen. In der Festschrift zum 60-jährigen Jubiläum des VVS wird der langjährige Verbandspräsident neben anderen früheren Gesinnungskollegen wie Ignaz Karl Gsur als Ehrenmitglied des Verbandes geführt. ${ }^{201}$ Gsur war wie Rhomberg einer der Verfechter des „Arierparagraphen“ im ÖSV. ${ }^{202}$

199 Vgl. Vernehmung des Beschuldigten Julius Funcke, Bezirksgericht Tamsweg, 21.11.1947, OÖLA, LG Linz Sondergerichte, Sch. 370, VgVr 6478/47.

200 Vgl. Melde- und Registrierungsblatt, OÖLA, LG Linz, Sondergerichte, Sch. 370, VgVr 6478/ 47; Salzburger Volkszeitung, 29.11.1946, S. 6.

201 Vgl. 60 Jahre Verband Vorarlberger Skiläufer. Von der Pionierzeit zur Weltmeisterschaft (Festschrift). Bregenz 1965, S. 12.

202 Vgl. Praher, Skifahren, S. 202-205. Andreas Praher, „Die Stadt gehörte wieder uns!“ Skilauf auf dem Bödele in nationalsozialistischen Zusammenhängen, in: Nikola Langreiter/Petra Zudrell (Hg.), Wem gehört das Bödele? Eine Kulturlandschaft verstehen, Salzburg/Wien 2020, S. 254-267, hier S. 265. 